University of Louisville ThinkIR: The University of Louisville's Institutional Repository

Electronic Theses and Dissertations

$8-2014$

\title{
Several functional equations defined on groups arising from stochastic distance measures.
}

Heather B. Hunt

University of Louisville

Follow this and additional works at: https://ir.library.louisville.edu/etd

Part of the Applied Mathematics Commons

\section{Recommended Citation}

Hunt, Heather B., "Several functional equations defined on groups arising from stochastic distance measures." (2014). Electronic Theses and Dissertations. Paper 2274.

https://doi.org/10.18297/etd/2274

This Doctoral Dissertation is brought to you for free and open access by ThinkIR: The University of Louisville's Institutional Repository. It has been accepted for inclusion in Electronic Theses and Dissertations by an authorized administrator of ThinkIR: The University of Louisville's Institutional Repository. This title appears here courtesy of the author, who has retained all other copyrights. For more information, please contact thinkir@louisville.edu. 


\title{
SEVERAL FUNCTIONAL EQUATIONS DEFINED ON GROUPS ARISING
} FROM STOCHASTIC DISTANCE MEASURES

\author{
By \\ Heather B. Hunt \\ B.S., University of Kentucky, May 2009 \\ M.A., University of Louisville, December 2011

\begin{abstract}
A Dissertation
Submitted to the Faculty of the

College of Arts and Sciences of the University of Louisville

in Partial Fulfillment of the Requirements

for the Degree of
\end{abstract}

Doctor of Philosophy

Department of Mathematics

University of Louisville

Louisville, KY

August 2014 

SEVERAL FUNCTIONAL EQUATIONS DEFINED ON GROUPS ARISING FROM STOCHASTIC DISTANCE MEASURES

Submitted by

Heather B. Hunt

A Dissertation Approved on

June 25, 2014

by the Following Reading and Examination Committee:

\author{
Dr. Prasanna K. Sahoo, Dissertation \\ Director
}

Dr. David Brown

Dr. Robert Powers

Dr. Thomas Riedel

Dr. Yongzhi Xu 


\section{ACKNOWLEDGEMENTS}

The five years that I have spent in graduate school have up to this point been the most educating, eye opening, and rewarding years of my life. I would have never made it through my Masters nor my Doctorate without the help and support of many people. It is to them that I owe my deepest gratitude.

We begin with where it all started, my students. Thank you to all of my students. You have helped motivate me to become a Professor, which in turn motivated me to get my $\mathrm{PhD}$ and complete this dissertation.

Thank you to all of my committee members: Dr. David Brown, Dr. Robert Powers, Dr. Thomas Riedel, Dr. Yongzhi Xu, and my advisor Dr. Prasanna Sahoo. I appreciate you taking the time to come watch my presentations, to read my dissertation, and for all of the useful feedback.

I would like to give a special thanks to Dr. Sahoo for taking on the challenge of being my advisor, the key word being challenge. As a fantastic educator, researcher, and family man I know that his plate is always full and yet somehow he managed to make time for me, constantly. I truly appreciate the dedication he has for his work and his students. Without his wisdom and guidance I would not be the mathematician that I am today. He was one of the best mentors one could ever ask for.

I would also like to give a special thanks to Dr. Riedel. He has been a wonderful boss, an outstanding mentor, and a valuable resource to myself and others. Dr. Riedel has helped me every step of the way on my journey as a graduate student; whether it be writing me a letter of recommendation, answering my millions 
of questions regarding all sort of mathematics, supporting my career decisions, or settling my nerves during times of uncertainty.

Lastly, I would like to thank my friends and family for their unwavering support, especially my fiancée Paul Elfen. How he deals with me day in and day out, I am not sure. As all graduate students know, graduate school is always challenging and is often very stressful. Thank you for helping me take my mind off of school and research every once in a while. Thank you for always listening to my stories, often unnecessary concerns, and complaints. I appreciate everything.

I have truly enjoyed the time that I have spent working on my dissertation and at the University of Louisville. I will always and forever be grateful for all of the people that I have met along this journey and the times that we have shared. I hope that all of the future journeys that I choose to embark on may be as fruitful and rewarding as this one has been.

Thank you! 


\section{ABSTRACT \\ SEVERAL FUNCTIONAL EQUATIONS DEFINED ON GROUPS ARISING FROM STOCHASTIC DISTANCE MEASURES}

\section{Heather B. Hunt}

June 25th, 2014

Several functional equations related to stochastic distance measures have been widely studied when defined on the real line. This dissertation generalizes several of those results to functions defined on groups and fields. Specifically, we consider when the domain is an arbitrary group, $G$, and the range is the field of complex numbers, $\mathbb{C}$.

We begin by looking at the linear functional equation $f(p r, q s)+f(p s, q r)=$ $2 f(p, q)+2 f(r, s)$ for all $p, q, r, s \in G$. The general solution $f: G \times G \rightarrow \mathbb{C}$ is given along with a few specific examples. Several generalizations of this equation are also considered and used to determine the general solution $f, g, h, k: G \times G \rightarrow \mathbb{C}$ of the functional equation $f(p r, q s)+g(p s, q r)=h(p, q)+k(r, s)$ for all $p, q, r, s \in G$.

We then consider the non-linear functional equation $f(p r, q s)+f(p s, q r)=$ $f(p, q) f(r, s)$. The solution $f: G \times G \rightarrow \mathbb{C}$ is given for all $p, q, r, s \in G$ when $f$ is an abelian function. It is followed by the structure of the general solution, $f$, dependent upon how the function acts on the center of the group. Several generalizations of the equation are also considered. The general structure of the solution $f, g, h$ : $G \times G \rightarrow \mathbb{C}$ of the functional equation $f(p r, q s)+f(p s, q r)=g(p, q) h(r, s)$ is given for all $p, q, r, s \in G$, dependent upon how the function $h$ acts on the center of the group. Future plans related to these equations will be given. 


\section{TABLE OF CONTENTS}

ACKNOWLEDGEMENTS ...................... iii

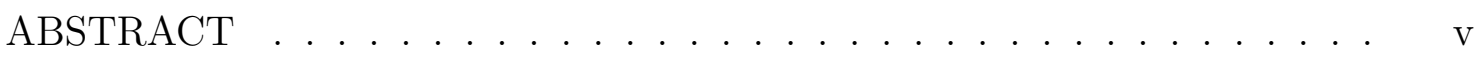
CHAPTER

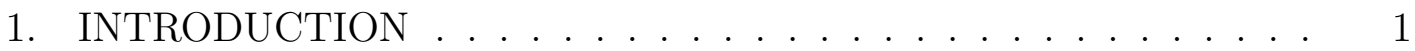

1.1 Introduction . . . . . . . . . . . . . . . . 1

1.2 Notation and Terminology . . . . . . . . . . . . . . 5

1.3 Examples of Central and Abelian Functions . . . . . . . 7

2. SEVERAL LINEAR FUNCTIONAL EQUATIONS ON GROUPS ARISING FROM STOCHASTIC DISTANCE MEASURES $\ldots \ldots \ldots$

2.1 Introduction: A Generalized Linear Functional Equation . . . . 10

2.2 Some Preliminary Results . . . . . . . . . . . . . . . . . . 11

2.3 The Soltuion of the Generalizaed Linear Functional . . . . . . 27

2.4 Several Examples Related to the Linear Functional Equation . . 31

3. A NON-LINEAR FUNCTIONAL EQUATION ON GROUPS ARISING FROM STOCHASTIC DISTANCE MEASURES . . . . . . . 35

3.1 Introduction: A Non-Linear Functional Equation . . . . . . . 35

3.2 Previous Results on A Related Functional Equation . . . . . . . 35

3.3 A Structure Theorem . . . . . . . . . . . . . . . . . . . . 36

4. SEVERAL GENERALIZATIONS OF A NON-LINEAR FUNCTIONAL EQUATION ON GROUPS ARISING FROM STOCHASTIC DISTANCE MEASURES . . . . . . . . . . . . . . . . . . . 42 
4.1 Introduction: Generalizations of A Non-Linear Functional Equa-

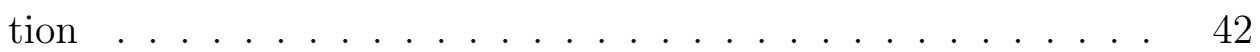

4.2 Generalization Theorems . . . . . . . . . . . . . . . 42

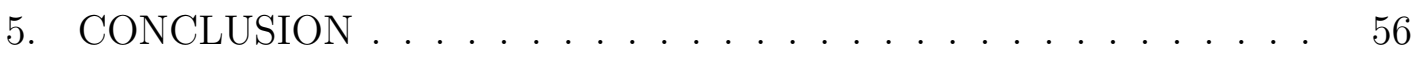

5.1 Conclusion and Future Plans . . . . . . . . . . . 56

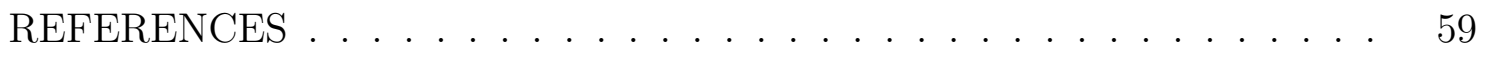

CURRICULUM VITAE . . . . . . . . . . . . . . . . . . . . 61 


\section{CHAPTER 1 INTRODUCTION}

\subsection{Introduction}

Let $\mathbb{R}$ denote the set of real numbers, $\mathbb{R}_{+}$the set of non-negative real numbers, $I$ the open unit interval $(0,1)$, and $I_{1}$ the open-closed unit interval $(0,1]$. Let $\Gamma_{n}^{0}$ be the set of discrete probability distributions on a finite set, $\Omega$ of cardinality $n$, that is

$$
\Gamma_{n}^{0}=\left\{P=\left(p_{1}, p_{2}, \ldots, p_{n}\right) \mid 0<p_{k}<1, \sum_{k=1}^{n} p_{k}=1\right\} .
$$

Several distance measures between discrete probability distributions have been proposed and studied over the past several years, such as the Chernoff coefficient, Hellinger coefficient, and Jeffereys' distance (see [2]). Notably, for $P$ and $Q$ in $\Gamma_{n}^{0}$, Kullback and Leibler in [7] defined a measure of the distance between $P$ and $Q$ that is both non-negative and non-symmetric. This measure, known as directed divergence, is defined as follows: for $P$ and $Q$ in $\Gamma_{n}^{0}$,

$$
D_{n}(P \| Q)=\sum_{k=1}^{n} p_{k} \log _{2} \frac{p_{k}}{q_{k}}
$$

Since it is neither symmetric nor satisfies the triangle inequality it is often not useful as a metric. In order to restore symmetry, the symmetric divergence between any two probability distributions, $P, Q$ in $\Gamma_{n}^{0}$, was introduced as follows:

$$
J_{n}(P, Q)=D_{n}(P \| Q)+D_{n}(Q \| P) .
$$


Explicitly, $J_{n}(P, Q)$ can be expressed as

$$
J_{n}(P, Q)=\sum_{k=1}^{n}\left(p_{k}-q_{k}\right) \log _{2} \frac{p_{k}}{q_{k}} .
$$

This measure, known as the J-divergence measure, satisfies the composition law

$$
J_{n m}(P \star R, Q \star S)+J_{n m}(P \star S, Q \star R)=2 J_{n}(P, Q)+2 J_{m}(R, S)
$$

for all $P, Q \in \Gamma_{n}^{0}$ and $R, S \in \Gamma_{m}^{0}$, where

$$
P \star R=\left(p_{1} r_{1}, \ldots, p_{1} r_{m}, p_{2} r_{1}, \ldots, p_{2} r_{m}, \ldots, p_{n} r_{1}, \ldots, p_{n} r_{m}\right) .
$$

Through this composition law and the sum property the measure has been characterized (see [2]).

Numerous well-known generalizations have also been characterized. One such generalization of the J-divergence measure being the symmetric divergence of degree $\alpha$ given by

$$
J_{n, \alpha}(P, Q)=\frac{\sum_{k=1}^{n}\left(p_{k}^{\alpha} q_{k}^{1-\alpha}+q_{k}^{\alpha} p_{k}^{1-\alpha}\right)-2}{2^{1-\alpha}-1},
$$

where $\alpha \neq 1$ and $P, Q$ in $\Gamma_{n}^{0}$. If $\alpha \rightarrow 1$, then $J_{n, \alpha}(P, Q)$ tends to $J_{n}(P, Q)$. This measure satisfies the following composition law [2]:

$$
\begin{aligned}
J_{n m, \alpha}(P \star R, Q \star S) & +J_{n m, \alpha}(P \star S, Q \star R) \\
& =2 J_{n, \alpha}(P, Q)+2 J_{m, \alpha}(R, S)+\lambda J_{n, \alpha}(P, Q) J_{m, \alpha}(R, S)
\end{aligned}
$$

for all $P, Q \in \Gamma_{n}^{0}$ and $R, S \in \Gamma_{m}^{0}$, where

$$
P \star R=\left(p_{1} r_{1}, \ldots, p_{1} r_{m}, p_{2} r_{1}, \ldots, p_{2} r_{m}, \ldots, p_{n} r_{1}, \ldots, p_{n} r_{m}\right) .
$$

and $\lambda=2^{1-\alpha}-1$. Through this composition law and the sum property the measure has been characterized (see [2]). 
Several functional equations were instrumental in both of these characterizations, notably

$$
f(p r, q s)+f(p s, q r)=(r+s) f(p, q)+(p+q) f(r, s)
$$

and

$$
f(p r, q s)+f(p s, q r)=f(p, q) f(r, s)
$$

for all $p, q, r, s \in I_{1}$ and $I$ respectively. These equations and numerous generalizations have been extensively studied when the unknown functions are defined on $I_{1}$ and $I$ and take values on the set $\mathbb{R}$.

One such generalization of the first equation is the following:

$$
f(p r, q s)+f(p s, q r)=g(r, s) f(p, q)+g(p, q) f(r, s)
$$

for all $p, q, r, s \in I_{1}$. The general solution of this functional equation was determined in [9] (see also [10]). In order to determine the general solution, the solution of the functional equation

$$
f(p r, q s)+f(p s, q r)=2 f(p, q)+2 f(r, s)
$$

for all $p, q, r, s \in I_{1}$ was needed. Riedel and Sahoo [10] have generalized this equation to

$$
f(p r, q s)+g(p s, q r)=h(p, q)+k(r, s)
$$

for all $p, q, r, s \in I_{1}$. For more information in regards to functional equations associated with stochastic distance measures, the interested reader is referred to the book [12].

A function $L: \mathbb{R}_{+} \rightarrow \mathbb{R}$ is said to be a logarithmic function if and only if $L(x y)=L(x)+L(y)$ for all $x, y \in \mathbb{R}_{+}$. A function $L: \mathbb{R}_{+} \times \mathbb{R}_{+} \rightarrow \mathbb{R}$ is called bi-logarithmic if and only if it is logarithmic in both variables, that is, $L(x y, w z)=$ $L(x, w z)+L(y, w z)=L(x, w)+L(x, z)+L(y, w)+L(y, z)$ for all $x, y, z, w \in \mathbb{R}_{+}$. 
A function $m: \mathbb{R}_{+} \rightarrow \mathbb{R}$ is said to be a multiplicative function if and only if $m(x y)=m(x) m(y)$ for all $x, y \in \mathbb{R}_{+}$.

In 1997, Riedel and Sahoo in [10] proved the following result on the openclosed unit interval.

THEOREM 1.1. The functions $f, g, h, k: I_{1}^{2} \rightarrow \mathbb{R}$ satisfy the functional equation

$$
f(p r, q s)+g(p s, q r)=h(p, q)+k(r, s)
$$

for all $p, q, r, s \in I_{1}$ if and only if

$$
\begin{aligned}
& f(p, q)=\mathcal{L}(p, q)+L_{2}(q)+\ell\left(\frac{p}{q}, \frac{p}{q}\right)+\phi(p q)-\alpha \\
& g(p, q)=\mathcal{L}(p, q)-L_{2}(q)+\ell\left(\frac{p}{q}, \frac{p}{q}\right)-\phi(p q)-\alpha \\
& h(p, q)=2 \mathcal{L}(p, q)+2 \ell\left(\frac{p}{q}, \frac{p}{q}\right)+2 \beta-2 \alpha \\
& k(p, q)=2 L_{1}(p)+2 L_{1}(q)+L_{2}(q)-L_{2}(p)+2 \ell\left(\frac{p}{q}, \frac{p}{q}\right)-2 \beta
\end{aligned}
$$

where

$$
\mathcal{L}(p, q)=L_{0}(q)-L_{0}(p)+L_{1}(p)+L_{1}(q)
$$

$L_{0}, L_{1}, L_{2}: \mathbb{R}_{+} \rightarrow \mathbb{R}$ are logarithmic, $\ell: \mathbb{R}_{+}^{2} \rightarrow \mathbb{R}$ is bi-logarithmic, $\phi: \mathbb{R}_{+} \rightarrow \mathbb{R}$ is arbitrary, and $\alpha, \beta$ are arbitrary real constants.

The open-closed unit interval, $I_{1}$, is a commutative unital semi-group under multiplication. The unit element 1 played a pivotal role in solving the above functional equation. In 1999, Sahoo in [11] gave a proof of Theorem 1.1 without using the unit element 1.

Far fewer generalizations of the second equation,

$$
f(p r, q s)+f(p s, q r)=f(p, q) f(r, s),
$$

have been studied. In 1989 Chung, Kannappan, Ng, and Sahoo (see [2] and also [12]) proved the following result on the open unit interval, $I$. The proof depends highly on the commutativity of the real number system on the open unit interval. 
THEOREM 1.2. Suppose $f: I^{2} \rightarrow \mathbb{R}$ satisfies the functional equation

$$
f(p r, q s)+f(p s, q r)=f(p, q) f(r, s)
$$

for all $p, q, r, s \in I$. Then

$$
f(p, q)=m_{1}(p) m_{2}(q)+m_{1}(q) m_{2}(p),
$$

where $m_{1}, m_{2}: I \rightarrow \mathbb{C}$ are multiplicative. Further, either $m_{1}$ and $m_{2}$ are both real or $m_{2}$ is the complex conjugate of $m_{1}$. The converse is also true.

In 2014, Stetkær (see [15]) studied a similar equation on a non-commutative semi-group, $S$. In the case that the semi-group is commutative, one can see that the result is similar to that of Chung, Kannappan, Ng, and Sahoo.

THEOREM 1.3. Let $S$ be a semi-group. Any solution $f: S \times S \rightarrow \mathbb{C}$ of the functional equation

$$
f(p r, q s)+f(s p, r q)=f(p, q) f(r, s)
$$

has the form

$$
f(p, q)=m_{1}(p) m_{2}(q)+m_{1}(q) m_{2}(p)
$$

for all $p, q, r, s \in S$, where $m_{1}, m_{2}: S \rightarrow \mathbb{C}$ are multiplicative.

Using several previous results along with new techniques and ideas, we will to extend several of these results to an arbitrary group.

\subsection{Notation and Terminology}

Let $(G, \cdot)$ and $(H, \star)$ be arbitrary groups. Let $e$ denote the identity element of any arbitrary group. A function $\theta: G \rightarrow H$ is said to be a homomorphism from group $G$ into group $H$ if and only if $\theta(x \cdot y)=\theta(x) \star \theta(y)$ for all $x, y \in G$. A function $\psi: G \times G \rightarrow H$ is said to be a bi-homomorphism if it is a homomorphism 
in each variable, that is, $\psi(x y, w z)=\psi(x, w z)+\psi(y, w z)=\psi(x, w)+\psi(x, z)+$ $\psi(y, w)+\psi(y, z)$ for all $x, y, z, w \in G$. A symmetric bi-homomorphism is a function $\psi: G \times G \rightarrow H$ that is a bi-homomorphism and satisfies $\psi(x, y)=\psi(y, x)$ for all $x, y \in G$.

If $G$ is an arbitrary group, then the group operation will be denoted by · and we write $x \cdot y$ simply as $x y$. In the case $G$ is an abelian group, the group operation will be denoted by addition. The set of all homomorphisms from $G$ into $H$ will be denoted by $\operatorname{Hom}(G, H)$. When the groups $G$ and $H$ are abelian, the homomorphism $\theta$ will be called an additive function and similarly $\psi$ will be called a bi-additive function. When the group $H$ is abelian, the homomorphism $\theta$ will be called an additive homomorphism and likewise $\psi$ will be called an additive bihomomorphism. When the groups $G$ and $H$ are both arbitrary the homomorphism $\theta$ will be called a multiplicative homomorphism and similarly $\psi$ will be called a multiplicative bi-homomorphism.

If $\mathbb{K}$ is a field, then the additive group of $\mathbb{K}$ will be denoted by $\mathbb{K}$ itself while the multiplicative group of $\mathbb{K}$ will be denoted by $\mathbb{K}^{\star}$. Specifically, we will denote the field of complex numbers by $\mathbb{C}$, and the field of non-zero complex number by $\mathbb{C}^{\star}$. Hence if $\mathbb{K}$ is a field, $\operatorname{Hom}(G, \mathbb{K})$ will denote the group of homomorphisms from the group $G$ to the additive group of the field $\mathbb{K}$, while $\operatorname{Hom}\left(G, \mathbb{K}^{\star}\right)$ will denote the group of homomorphisms from the group $G$ to the multiplicative group of the field $\mathbb{K}$. The set of all bi-homomorphisms from $G \times G$ into the additive group of the field $\mathbb{K}$ will be denoted by $\operatorname{Bihom}(G \times G, \mathbb{K})$, while the set of all symmetric bi-homomorphisms will be denoted by $\operatorname{SBihom}(G \times G, \mathbb{K})$. It should be noted that $\operatorname{SBihom}(G \times G, \mathbb{K}) \subseteq \operatorname{Bihom}(G \times G, \mathbb{K})$.

A function $f: G \rightarrow \mathbb{K}$ is said to be a central function if and only if $f(x y)=$ $f(y x)$ for all $x, y \in G$. A central function is a function invariant under conjugation. A function $f: G \rightarrow \mathbb{K}$ is said to be an abelian function if and only if $f(x y z)=f(x z y)$ 
for all $x, y, z \in G$ [14]. It is easy to see that every abelian function is central while the converse is not true. An element $f \in \operatorname{Hom}\left(G, \mathbb{C}^{\star}\right)$ is called a (group) character. Hence a character is a non-zero multiplicative homomorphism from group $G$ into multiplicative group of non-zero complex numbers. For any function $f$ we will use the following notation: $f_{x}(y)=f(x y)-f(x) f(y)$ for all $x, y \in G$.

\subsection{Examples of Central and Abelian Functions}

In this section we give an example of a central function and an abelian function.

EXAMPLE 1.1. Consider the non-abelian group of $2 \times 2$ invertible matrices over $\mathbb{R}$

$$
G L_{2}(\mathbb{R})=\left\{\left[\begin{array}{ll}
a & b \\
c & d
\end{array}\right] \mid a, b, c, d \in \mathbb{R}, a d-b c \neq 0\right\} .
$$

Consider the function $f: G L_{2}(\mathbb{R}) \rightarrow \mathbb{R}$ defined such that $f(A)=$ ad $-b c=\operatorname{det}(A)$ for all $\in G L_{2}(\mathbb{R})$. It is known that the following holds for all $A, B \in G L_{2}(\mathbb{R})$ :

$$
\operatorname{det}(A B)=\operatorname{det}(A) \operatorname{det}(B)=\operatorname{det}(B) \operatorname{det}(A)=\operatorname{det}(B A) .
$$

Therefore, $f(A B)=f(B A)$ although $A B \neq B A$.

One can easily see that this function is also an abelian function by the properties of the real numbers and the determinant. We will now give a more complex example of an abelian function.

EXAMPLE 1.2. Consider the symmetric permutation group of order 6 , that is the group $S_{3}=\{e,(12),(13),(23),(123),(132)\}$. This group is a non-abelian group, for instance $(12)(13)=(132)$ but $(13)(12)=(123)$. It can be shown that the commutator subgroup, which is the set of elements given by $a b a^{-1} b^{-1}$ where $a, b \in S_{3}$, 
is the set $A_{3}$. The set $A_{3}$ consists of the identity element and the two 3-cycles, i.e. the set $S_{3}^{\prime}=\left\{a b a^{-1} b^{-1} \mid a, b \in S_{3}\right\}=\{e,(123),(132)\}=A_{3}$.

We consider the abelianization of $S_{3}$, which is the quotient group $S_{3} / A_{3}=$ $\left\{a A_{3} \mid a \in S_{3}\right\}$. The abelianization creates a partition of the set $S_{3}$. The subsets within this partition are called the cosets. We have that there are two cosets: the set of all three 2-cycles and the set with the identity and the two 3-cycles. If we define a function that is constant on the cosets, i.e. $f(x y)=f(x)$ for all $x \in S_{3}$ and $y \in S_{3}^{\prime}$, then we have created an abelian function. Thus, consider the function $f: S_{3} \rightarrow \mathbb{R}$ defined as follows:

$$
f(x)= \begin{cases}c_{1} & \text { if } x \in\{(12),(13),(23)\} \\ c_{2} & \text { if } x \in\{e,(123),(132)\}\end{cases}
$$

where $c_{1}$ and $c_{2}$ are arbitrary real constants.

It is a well known that every permutation in $S_{n}$, for an arbitrary $n$, can be broken down into a product of 2-cycles. In terms of $S_{3}$ the set $\{(12),(13),(23)\}$ is the set of all odd permutations which are permutations that can be broken down into an odd number of two cycles. The set $\{e,(123),(132)\}$ is the set of all even permutations which are permutations that can be broken down into an even number of two cycles. The set of all even permutations is denoted $A_{n}$.

If we consider multiplying any three elements of $S_{3}$ then the number of two cycles that the product breaks down into will determine whether or not the product is an even or odd permutation which will determine the value of our function. Thus, if we consider three elements $x, y, z \in S_{3}$ the function $f$ will take the same value for any permutation of these three elements. Therefore, this function is an abelian function.

REMARK 1.1. The previous example can be generalized for any integer $n \geq 3$. It is known that the commutator subgroup for any symmetric group is the group of 
even permutations, $A_{n}$. The quotient group $S_{n} / A_{n}$ will always produce two cosets. Hence, you can always create a function just like the one from the previous example.

Since every central function is not necessarily abelian, it is only fitting to give an example of such a function.

EXAMPLE 1.3. Consider the non-abelian group of $2 \times 2$ invertible matrices over $\mathbb{R}$,

$$
G L_{2}(\mathbb{R})=\left\{\left[\begin{array}{ll}
a & b \\
c & d
\end{array}\right] \mid a, b, c, d \in \mathbb{R}, a d-b c \neq 0\right\},
$$

and the function $f: G L_{2}(\mathbb{R}) \rightarrow \mathbb{R}$ defined such that $f(A)=$ trace $(A)$ for all $A \in$ $G L_{2}(\mathbb{R})$, where the trace represents the sum of the elements on the main diagonal, i.e. $a+d$. It can be shown that the following holds for all $A, B \in G L_{2}(\mathbb{R})$ :

$$
\operatorname{trace}(A B)=\operatorname{trace}(B A) .
$$

Therefore, $f(A B)=f(B A)$ although $A B \neq B A$. It is not necessarily true that $\operatorname{trace}(A B C)=\operatorname{trace}(A C B)$ for all $A, B, C \in G L_{2}(\mathbb{R})$. Since $f(A B C)$ is not always equal to $f(A C B)$, we have that $f$ is central but not abelian. 
CHAPTER 2

\section{SEVERAL LINEAR FUNCTIONAL EQUATIONS ON GROUPS ARISING FROM STOCHASTIC DISTANCE MEASURES}

\subsection{Introduction: A Generalized Linear Functional Equation}

The main goal of this Chapter is to find the general solution $f, g, h, k$ : $G \times G \rightarrow \mathbb{C}$ of the linear functional equation

$$
f(p r, q s)+g(p s, q r)=h(p, q)+k(r, s)
$$

on an arbitrary group. Here $G$ denotes an arbitrary group and $\mathbb{C}$ denotes the field of complex numbers. In order to achieve this task, we also determine the general solution of three important functional equations, namely,

$$
\begin{gathered}
f(p r, q s)+f(p s, q r)=2 f(p, q)+2 f(r, s) \\
f(p r, q s)+f(p s, q r)=2 f(p, q)+f(r, s)+f(s, r)
\end{gathered}
$$

and

$$
f(p r, q s)-f(p s, q r)=f(r, s)-f(s, r)
$$

for all $p, q, r, s \in G$. For the functional equation (2.1), no new solutions emerge in this general setting. We will give several specific examples of solutions to the first of the three equations given above.

One should note that the work within this Chapter has been accepted for publication in the journal Aequationes Mathematicae, under the title "On a functional equation on groups arising from the characterization of stochastic distance measures" [5]. 


\subsection{Some Preliminary Results}

We begin with an important result concerning the symmetrized homomorphism equation due to Corovei (see [3] and also [14]).

THEOREM 2.1. Let $G$ be a group and $\mathbb{K}$ be a field with characteristic not equal to

2. The function $f: G \rightarrow \mathbb{K}$ satisfies the functional equation

$$
f(x y)+f(y x)=2 f(x)+2 f(y) \quad \forall x, y \in G
$$

if and only if $f \in \operatorname{Hom}(G, \mathbb{K})$.

First, we determine the general solution of the functional equation

$$
f(p r, q s)+f(p s, q r)=2 f(p, q)+2 f(r, s)
$$

on an arbitrary group $G$ using some ideas from [2] and Theorem 2.1.

THEOREM 2.2. The function $f: G \times G \rightarrow \mathbb{C}$ satisfies the functional equation (2.2) for all $p, q, r, s \in G$ if and only if $f$ is of the form

$$
f(p, r)=\theta(p)+\theta(r)+\psi\left(p r^{-1}, p r^{-1}\right)
$$

where $\theta \in \operatorname{Hom}(G, \mathbb{C})$ and $\psi \in \operatorname{SBihom}(G \times G, \mathbb{C})$.

Proof. It is easy to verify that $f$ given by (2.4) is a solution of the equation (2.2). Hence, we only prove the converse. Let $r=s=e$ in (2.2). Thus,

$$
f(p, q)+f(p, q)=2 f(p, q)+2 f(e, e)
$$

giving us $f(e, e)=0$. Switching $r$ and $s$ in (2.2) we find that

$$
f(p s, q r)+f(p r, q s)=2 f(p, q)+2 f(s, r) .
$$

Subtracting this from (2.2) yields

$$
f(r, s)=f(s, r)
$$


for all $r, s \in G$, thus $f$ is symmetric. Now, let $q=s=e$ in (2.2). Then

$$
f(p r, e)+f(p, r)=2 f(p, e)+2 f(r, e)
$$

for all $p, r \in G$. Define a function $g: G \rightarrow \mathbb{C}$ such that $g(x)=f(x, e)$ for all $x \in G$. Since $f(e, e)=0$ we have that $g(e)=0$. Using the definition of $g$ the last equation yields

$$
f(p, r)=2 g(p)+2 g(r)-g(p r)
$$

Since $f$ is symmetric, we can see that

$$
g(r p)=g(p r)
$$

for all $p, r \in G$. Thus, $g$ is a central function. Now, let $s=e$ in (2.2) to obtain

$$
f(p r, q)+f(p, q r)=2 f(p, q)+2 f(r, e) .
$$

Using the definition of $g$, the fact that $g$ is a central function, and (2.4) the previous becomes

$$
\begin{aligned}
2 g(p r)+2 g(q)-g(p r q)+2 g(p) & +2 g(q r)-g(p q r) \\
& =4 g(p)+4 g(q)-2 g(p q)+2 g(r)
\end{aligned}
$$

and simplifies to

$$
\begin{aligned}
g(p r q)-g(p)-g(r q) & +g(p q r)-g(p)-g(q r) \\
& =2 g(p r)-2 g(p)-2 g(r)+2 g(p q)-2 g(p)-2 g(q) .
\end{aligned}
$$

For a fixed $p \in G$ we define $\omega: G \rightarrow \mathbb{C}$ such that

$$
\omega(r)=g(p r)-g(p)-g(r)
$$

Thus, (2.5) becomes

$$
\omega(r q)+\omega(q r)=2 \omega(r)+2 \omega(q)
$$


for all $q, r \in G$. By Theorem 2.1 we have that $\omega$ is an additive homomorphism. Since $\omega$ was defined for each fixed $p, \omega$ can be written as $\omega(r)=2 \psi(p, r)$, where $\psi: G \times G \rightarrow \mathbb{C}$ is an additive homomorphism in the second variable. Using (2.6) we get

$$
g(p r)-g(p)-g(r)=2 \psi(p, r)
$$

for all $p, r \in G$. Interchanging $r$ with $p$ we get

$$
g(r p)-g(r)-g(p)=2 \psi(r, p)
$$

Comparing the two previous equations and using the fact that $g$ is a central function we find that $\psi$ is a symmetric function. Hence, $\psi$ is an additive homomorphism in both variables and $\psi \in \operatorname{SBihom}(G \times G, \mathbb{C})$. Now, define $\theta: G \rightarrow \mathbb{C}$ such that

$$
\theta(p)=g(p)-\psi(p, p)
$$

for all $p \in G$. Then

$$
g(p r)=\theta(p r)+\psi(p r, p r)
$$

for all $p, r \in G$. Since $\psi$ is a symmetric bi-homomorphism, expanding $\psi(p r, p r)$ we get

$$
\psi(p r, p r)=\psi(p, p)+\psi(r, r)+\psi(p, r)+\psi(r, p)
$$

Hence from $(2.7),(2.9)$, and the symmetry of $\psi$ we get

$$
\begin{array}{r}
2 \psi(p, r)=\theta(p r)+\psi(p r, p r)-\theta(p)-\psi(p, p)-\theta(r)-\psi(r, r) \\
=\theta(p r)+\psi(p, p)+\psi(r, r)+\psi(p, r)+\psi(r, p) \\
-\theta(p)-\psi(p, p)-\theta(r)-\psi(r, r)
\end{array}
$$

which simplifies to

$$
\theta(p r)=\theta(p)+\theta(r)
$$


for all $p, r \in G$. Thus, $\theta$ is an additive homomorphism. Substituting (2.8) into (2.4) and using (2.9) we find that

$$
\begin{aligned}
f(p, r) & =2(\theta(p)+\psi(p, p))+2(\theta(r)+\psi(r, r))-(\theta(p r)+\psi(p r, p r)) \\
& =\theta(p)+\theta(r)+2 \psi(p, p)+2 \psi(r, r)-\psi(p r, p r) \\
& =\theta(p)+\theta(r)+2 \psi(p, p)+2 \psi(r, r)-\psi(p, p)-\psi(r, r)-\psi(p, r)-\psi(r, p) \\
& =\theta(p)+\theta(r)+\psi(p, p)+\psi\left(r^{-1}, r^{-1}\right)+\psi\left(p, r^{-1}\right)+\psi\left(r^{-1}, p\right) \\
& =\theta(p)+\theta(r)+\psi\left(p r^{-1}, p r^{-1}\right) .
\end{aligned}
$$

Hence, $f(p, r)=\theta(p)+\theta(r)+\psi\left(p r^{-1}, p r^{-1}\right)$ for all $p, r \in G$ which is the asserted solution (2.4). Now the proof of the theorem is complete.

This theorem leads us to several generalizations of the equation in terms of two functions, hence the following corollaries.

COROLLARY 2.1. The functions $f, g: G \times G \rightarrow \mathbb{C}$ satisfy the functional equation

$$
f(p r, q s)+g(p s, q r)=2 f(p, q)+2 f(r, s)
$$

for all $p, q, r, s \in G$ if and only if $f$ and $g$ are of the form

$$
\begin{aligned}
& f(p, r)=\theta(p)+\theta(r)+\psi\left(p r^{-1}, p r^{-1}\right)+\alpha \\
& g(p, r)=\theta(p)+\theta(r)+\psi\left(p r^{-1}, p r^{-1}\right)+3 \alpha
\end{aligned}
$$

where $\theta \in \operatorname{Hom}(G, \mathbb{C}), \psi \in \operatorname{SBihom}(G \times G, \mathbb{C})$, and $\alpha \in \mathbb{C}$ is an arbitrary constant.

Proof. Let $r=s=e$ in (2.10). Thus,

$$
f(p, q)+g(p, q)=2 f(p, q)+2 f(e, e) .
$$

Let $\alpha$ be a constant in $\mathbb{C}$ such that $\alpha=f(e, e)$. The last equation then becomes

$$
g(p, q)=f(p, q)+2 \alpha
$$


for all $p, q \in G$. Substituting this back into (2.10) and rewriting we have

$$
h(p r, q s)+h(p s, q r)=2 h(p, q)+2 h(r, s)
$$

where $h: G \times G \rightarrow \mathbb{C}$ is defined by $h(p, q)=f(p, q)-\alpha$ for all $p, q \in G$. From Theorem 2.2 we now have the following solution:

$$
h(p, r)=\theta(p)+\theta(r)+\psi\left(p r^{-1}, p r^{-1}\right)
$$

for all $p, r \in G$, where $\theta: G \rightarrow \mathbb{C}$ is an additive homomorphism and $\psi: G \times G \rightarrow \mathbb{C}$ is an additive symmetric bi-homomorphism. Therefore, from the definition of $h$ we get

$$
f(p, r)=\theta(p)+\theta(r)+\psi\left(p r^{-1}, p r^{-1}\right)+\alpha
$$

for all $p, r \in G$. Thus,

$$
g(p, r)=\theta(p)+\theta(r)+\psi\left(p r^{-1}, p r^{-1}\right)+3 \alpha
$$

for all $p, r \in G$.

It is easy to check that the functions $f$ and $g$ given above are indeed the solutions of the equation (2.10) and now the proof of the corollary is complete.

COROLlARY 2.2. If $f, g: G \times G \rightarrow \mathbb{C}$ satisfy the following functional equation

$$
f(p r, q s)+g(p s, q r)=2 f(p, q)+2 g(r, s)
$$

for all $p, q, r, s \in G$, then $f$ and $g$ are of the form

$$
\begin{aligned}
& f(p, r)=\theta(p)+\theta(r)+\psi\left(p r^{-1}, p r^{-1}\right)-\alpha \\
& g(p, r)=\theta(p)+\theta(r)+\psi\left(p r^{-1}, p r^{-1}\right)+\alpha
\end{aligned}
$$

where $\theta \in \operatorname{Hom}(G, \mathbb{C}), \psi \in \operatorname{SBihom}(G \times G, \mathbb{C})$, and $\alpha \in \mathbb{C}$ is an arbitrary constant. The converse of this is also true. 
Proof. Let $r=s=e$ in (2.2). Thus,

$$
f(p, q)+g(p, q)=2 f(p, q)+2 g(e, e)
$$

for all $p, q \in G$. Let $\alpha$ be in $\mathbb{C}$ such that $\alpha=g(e, e)$. The previous gives us

$$
g(p, q)=f(p, q)+2 \alpha
$$

for all $p, q \in G$. Substituting this back into (2.11) and rewriting we have

$$
h(p r, q s)+h(p s, q r)=2 h(p, q)+2 h(r, s),
$$

where $h: G \times G \rightarrow \mathbb{C}$ is defined by $h(p, q)=f(p, q)+\alpha$ for all $p, q \in G$. From Theorem 2.2 we now have the following solution of the last functional equation:

$$
h(p, r)=\theta(p)+\theta(r)+\psi\left(p r^{-1}, p r^{-1}\right)
$$

for all $p, r \in G$, where $\theta: G \rightarrow \mathbb{C}$ is an additive homomorphism and $\psi: G \times G \rightarrow \mathbb{C}$ is an additive symmetric bi-homomorphism. Therefore, from the definition of $h$

$$
f(p, r)=\theta(p)+\theta(r)+\psi\left(p r^{-1}, p r^{-1}\right)-\alpha
$$

for all $p, r \in G$. Thus,

$$
g(p, r)=\theta(p)+\theta(r)+\psi\left(p r^{-1}, p r^{-1}\right)+\alpha
$$

for all $p, r \in G$.

The converse is easy to verify and hence the proof of the corollary is now complete.

COROLlaRY 2.3. The functions $f, g: G \times G \rightarrow \mathbb{C}$ satisfy the functional equation

$$
f(p r, q s)+f(p s, q r)=2 g(p, q)+2 g(r, s)
$$

for all $p, q, r, s \in G$ if and only if $f$ and $g$ are of the form

$$
\begin{aligned}
& f(p, q)=\theta(p)+\theta(q)+\psi\left(p q^{-1}, p q^{-1}\right)+2 \alpha \\
& g(p, q)=\theta(p)+\theta(r)+\psi\left(p r^{-1}, p r^{-1}\right)+\alpha
\end{aligned}
$$

where $\theta \in \operatorname{Hom}(G, \mathbb{C}), \psi \in \operatorname{SBihom}(G \times G, \mathbb{C})$, and $\alpha \in \mathbb{C}$ is an arbitrary constant. 
Proof. Let $r=s=e$ in (2.12). We then have the following:

$$
2 f(p, q)=2 g(p, q)+2 g(e, e) .
$$

Let $\alpha \in \mathbb{C}$ be a constant such that $\alpha=g(e, e)$. Thus, we have

$$
2 g(p, q)=2 f(p, q)-2 \alpha
$$

for all $p, q \in G$. Substituting this back into (2.12) we get

$$
f(p r, q s)+f(p s, q r)=2 f(p, q)-2 \alpha+2 f(r, s)-2 \alpha
$$

for all $p, q, r, s \in G$. Now, defining a function $h: G \times G \rightarrow \mathbb{C}$ such that $h(p, q)=$ $f(p, q)-2 \alpha$ for all $p, q \in G$, the previous equation reduces to

$$
h(p r, q s)+h(p s, q r)=2 h(p, q)+2 h(r, s)
$$

for all $p, q, r, s \in G$. Thus by Theorem 2.2 and the fact that $f(p, q)=h(p, q)+2 \alpha$ and $g(p, q)=f(p, q)-\alpha$ we have the following:

$$
\begin{aligned}
& h(p, q)=\theta(p)+\theta(q)+\psi\left(p q^{-1}, p q^{-1}\right), \\
& f(p, q)=\theta(p)+\theta(q)+\psi\left(p q^{-1}, p q^{-1}\right)+2 \alpha, \\
& g(p, q)=\theta(p)+\theta(r)+\psi\left(p r^{-1}, p r^{-1}\right)+\alpha
\end{aligned}
$$

for all $p, q \in G$, where $\theta: G \rightarrow \mathbb{C}$ is an additive homomorphism and $\psi: G \times G \rightarrow \mathbb{C}$ is an additive symmetric bi-homomorphism. This completes the proof.

So far we have been working with functional equations in which the more prevalent function $f$ is symmetric. We must now consider a generalization of the functional equation (2.2) when the unknown function is not symmetric.

THEOREM 2.3. The function $f: G \times G \rightarrow \mathbb{C}$ satisfies the functional equation

$$
f(p r, q s)+f(p s, q r)=2 f(p, q)+f(r, s)+f(s, r)
$$


for all $p, q, r, s$ in $G$ if and only if $f$ is of the form

$$
f(p, q)=\theta_{1}(p)+\theta_{1}(q)-\theta_{2}(p)+\theta_{2}(q)+\psi\left(p q^{-1}, p q^{-1}\right),
$$

where $\theta_{1}, \theta_{2} \in \operatorname{Hom}(G, \mathbb{C})$ and $\psi \in \operatorname{SBihom}(G \times G, \mathbb{C})$.

Proof. The only if part of the theorem is easy to verify. Thus, we only prove the if part of the theorem. Let $s=q=e$ in (2.13). Then we have

$$
f(p, r)=2 f(p, e)+f(r, e)+f(e, r)-f(p r, e)
$$

for all $p, r \in G$. Now, define two functions $g, h: G \rightarrow \mathbb{C}$ such that for all $p \in G$

$$
g(p)=f(p, e) \text { and } h(p)=f(e, p) .
$$

Thus, (2.14) becomes

$$
f(p, r)=2 g(p)+g(r)+h(r)-g(p r)
$$

for all $p, r \in G$. Substituting $p=r=e$ in (2.13) we get

$$
f(s, q)=2 f(e, q)+f(e, s)+f(s, e)-f(e, q s)
$$

for all $q, s \in G$. Using the definitions of $g$ and $h(2.16)$ reduces to

$$
f(s, q)=2 h(q)+h(s)+g(s)-h(q s) .
$$

If we consider $s=p$ and $q=r$ in the previous we have

$$
f(p, r)=2 h(r)+h(p)+g(p)-h(r p)
$$

for all $p, r \in G$. Comparing (2.15) and (2.17) and simplifying the resulting expression, we find that

$$
h(r p)-g(p r)=h(r)+h(p)-g(p)-g(r)
$$


for all $p, r \in G$. Interchanging $p$ and $r$ in (2.18) yields

$$
h(p r)-g(r p)=h(p)+h(r)-g(r)-g(p) .
$$

Now, adding (2.19) to (2.18) we get the following:

$$
h(r p)-g(p r)+h(p r)-g(r p)=2 h(r)+2 h(p)-2 g(p)-2 g(r)
$$

for all $p, r \in G$. Define a function $\theta_{1}: G \rightarrow \mathbb{C}$ such that $\theta_{1}(p)=h(p)-g(p)$ for all $p \in G$, then the previous becomes

$$
\theta_{1}(r p)+\theta_{1}(p r)=2 \theta_{1}(p)+2 \theta_{1}(r)
$$

for all $p, r \in G$. By Theorem 2.1 (see [3]) we have that $\theta_{1}$ is an additive homomorphism. Therefore,

$$
\theta_{1}(p r)=\theta_{1}(p)+\theta_{1}(r)
$$

and using the definition of $\theta_{1}$, we see that

$$
h(p r)-g(p r)=h(p)-g(p)+h(r)-g(r) .
$$

Comparing the last equality with (2.19) we have

$$
g(p r)=g(r p)
$$

for all $p, r \in G$. Similarly, using (2.15) we find that $h(p r)=h(r p)$. Thus, both $g$ and $h$ are central functions. Now, by the way $\theta_{1}$ was defined we get

$$
h(p)=g(p)+\theta_{1}(p)
$$

for all $p \in G$. Using this in (2.14) yields

$$
f(p, r)=2 g(p)+2 g(r)+\theta_{1}(r)-g(p r)
$$

Interchanging $p$ with $r$ in the last equation, we obtain

$$
f(r, p)=2 g(r)+2 g(p)+\theta_{1}(p)-g(r p)
$$


Now, subtracting and using the fact that $g$ is a central function we find that

$$
f(p, r)-f(r, p)=\theta_{1}(r)-\theta_{1}(p)
$$

for all $p, r \in G$. Using this in (2.13) we get the following:

$$
f(p r, q s)+f(p s, q r)=2 f(p, q)+2 f(r, s)+\theta_{1}(r)-\theta_{1}(s)
$$

for all $p, q, r, s \in G$. Since $\theta_{1}$ is an additive homomorphism we have that $2 \theta_{1}$ is also an additive homomorphism. Thus, we can define $\theta_{2}: G \rightarrow \mathbb{C}$ such that $2 \theta_{2}(p)=\theta_{1}(p)$ for all $p \in G$ where $\theta_{2}$ is an additive homomorphism. The last expression now becomes

$$
f(p r, q s)+f(p s, q r)=2 f(p, q)+2 f(r, s)+2 \theta_{2}(r)-2 \theta_{2}(s) .
$$

Defining a function $k: G \times G \rightarrow \mathbb{C}$ such that $k(p, q)=f(p, q)+\theta_{2}(p)-\theta_{2}(q)$ for all $p, q \in G$, the previous expression becomes

$$
k(p r, q s)+k(p s, q r)=2 k(p, q)+2 k(r, s)
$$

for all $p, q, r, s \in G$. This equation was solved in Theorem 2.2. Therefore, we have that

$$
k(p, q)=\theta(p)+\theta(q)+\psi\left(p q^{-1}, p q^{-1}\right)
$$

for all $p, q \in G$, where $\theta: G \rightarrow \mathbb{C}$ is an additive homomorphism and $\psi: G \times G \rightarrow \mathbb{C}$ is an additive symmetric bi-homomorphism. Therefore, using the definition of $k$ we have

$$
f(p, q)=\theta(p)+\theta(q)-\theta_{2}(p)+\theta_{2}(q)+\psi\left(p q^{-1}, p q^{-1}\right),
$$

where $\theta$ and $\theta_{2}$ are additive homomorphisms and $\psi$ is an additive symmetric bihomomorphism. This completes the proof of the theorem. 
COROLLARY 2.4. The functions $f, g: G \times G \rightarrow \mathbb{C}$ satisfy the functional equation

$$
f(p r, q s)+f(p s, q r)=2 f(p, q)+2 g(r, s)
$$

for all $p, q, r, s \in G$ if and only if $f$ and $g$ are of the form

$$
\begin{aligned}
& f(p, q)=\theta_{1}(p)+\theta_{1}(q)-\theta_{2}(p)+\theta_{2}(q)+\psi\left(p q^{-1}, p q^{-1}\right)+\alpha \\
& g(p, q)=\theta_{1}(p)+\theta_{1}(q)+\psi\left(p q^{-1}, p q^{-1}\right),
\end{aligned}
$$

where $\theta_{1}, \theta_{2} \in \operatorname{Hom}(G, \mathbb{C}), \psi \in \operatorname{SBihom}(G \times G, \mathbb{C})$, and $\alpha \in \mathbb{C}$ is an arbitrary constant.

Proof. It is easy to see that $f$ and $g$ as asserted in the corollary satisfy the functional equation (2.20). So, we only prove the converse. Let $p=q=e$ in (2.20). We get

$$
f(r, s)+f(s, r)=2 f(e, e)+2 g(r, s)
$$

for all $r, s \in G$. Let $\alpha \in \mathbb{C}$ be a constant such that $\alpha=f(e, e)$. Then, the previous becomes

$$
2 g(r, s)=f(r, s)+f(s, r)-2 \alpha
$$

for all $r, s \in G$. Substituting this into (2.20) we have the following

$$
f(p r, q s)+f(p s, q r)=2 f(p, q)+f(r, s)+f(s, r)-2 \alpha
$$

for all $p, q, r, s \in G$. Defining a function $h: G \times G \rightarrow \mathbb{C}$ such that $h(p, q)=f(p, q)-\alpha$ for all $p, q \in G$, the previous equation reduces to

$$
h(p r, q s)+h(p s, q r)=2 h(p, q)+h(r, s)+h(s, r) .
$$

From Theorem 2.3 we obtain

$$
h(p, q)=\theta_{1}(p)+\theta_{1}(q)-\theta_{2}(p)+\theta_{2}(q)+\psi\left(p q^{-1}, p q^{-1}\right),
$$


where $\theta_{1}, \theta_{2}: G \rightarrow \mathbb{C}$ are additive homomorphisms and $\psi: G \times G \rightarrow \mathbb{C}$ is an additive symmetric bi-homomorphism. Therefore, using the definition of $h$, we have

$$
f(p, q)=\theta_{1}(p)+\theta_{1}(q)-\theta_{2}(p)+\theta_{2}(q)+\psi\left(p q^{-1}, p q^{-1}\right)+\alpha
$$

for all $p, q \in G$. Now substituting this back into (2.21) and simplifying we get the following:

$$
g(p, q)=\theta_{1}(p)+\theta_{1}(q)+\psi\left(p q^{-1}, p q^{-1}\right)
$$

for all $p, q \in G$ and the proof of the corollary is now complete.

COROLLARY 2.5. If $f, g, h: G \times G \rightarrow \mathbb{C}$ satisfy the following functional equation

$$
f(p r, q s)+f(p s, q r)=2 g(p, q)+2 h(r, s)
$$

for all $p, q, r, s \in G$, then $f, g$ and $h$ are of the form

$$
\begin{aligned}
& f(p, q)=\theta_{1}(p)+\theta_{1}(q)-\theta_{2}(p)+\theta_{2}(q)+\psi\left(p q^{-1}, p q^{-1}\right)+\beta \\
& g(p, q)=\theta_{1}(p)+\theta_{1}(q)-\theta_{2}(p)+\theta_{2}(q)+\psi\left(p q^{-1}, p q^{-1}\right)+\beta-\alpha \\
& h(p, q)=\theta_{1}(p)+\theta_{1}(q)+\psi\left(p q^{-1}, p q^{-1}\right)+\alpha,
\end{aligned}
$$

where $\theta_{1}, \theta_{2} \in \operatorname{Hom}(G, \mathbb{C}), \psi \in \operatorname{SBihom}(G \times G, \mathbb{C})$, and $\alpha$ and $\beta$ are arbitrary constants in $\mathbb{C}$. The converse is also true.

Proof. Let $r=s=e$ in (2.22). Then we have the following:

$$
2 f(p, q)=2 g(p, q)+2 h(e, e) .
$$

Let $\alpha \in G$ be a constant such that $\alpha=h(e, e)$, the previous then becomes

$$
2 g(p, q)=2 f(p, q)-2 \alpha
$$

for all $p, q \in G$. Now, substituting back into (2.22) we get

$$
f(p r, q s)+f(p s, q r)=2 f(p, q)-2 \alpha+2 h(r, s) .
$$


Defining a function $k: G \times G \rightarrow \mathbb{C}$ such that $k(p, q)=h(p, q)-\alpha$ for all $p, q \in G$, the previous reduces to

$$
f(p r, q s)+f(p s, q r)=2 f(p, q)+2 k(r, s)
$$

for all $p, q, r, s \in G$. Using Corollary 2.4, we have

$$
\begin{aligned}
& f(p, q)=\theta_{1}(p)+\theta_{1}(q)-\theta_{2}(p)+\theta_{2}(q)+\psi\left(p q^{-1}, p q^{-1}\right)+\beta \\
& k(p, q)=\theta_{1}(p)+\theta_{1}(q)+\psi\left(p q^{-1}, p q^{-1}\right)
\end{aligned}
$$

where $\theta_{1}, \theta_{2}: G \rightarrow \mathbb{C}$ are an additive homomorphisms, $\psi: G \times G \rightarrow \mathbb{C}$ is an additive symmetric bi-homomorphism, and $\beta$ is an arbitrary constant in $\mathbb{C}$. Therefore,

$$
h(p, q)=\theta_{1}(p)+\theta_{1}(q)+\psi\left(p q^{-1}, p q^{-1}\right)+\alpha
$$

for all $p, q \in G$. Finally, using (2.23) and (2.24) we have that

$$
g(p, q)=\theta_{1}(p)+\theta_{1}(q)+\psi\left(p q^{-1}, p q^{-1}\right)-\theta_{2}(p)+\theta_{2}(q)+\beta-\alpha
$$

for all $p, q \in G$. The converse is easy to show and now the proof is complete.

COROLlARY 2.6. The functions $f, g, h: G \times G \rightarrow \mathbb{C}$ satisfy the functional equation

$$
f(p r, q s)+g(p s, q r)=2 f(p, q)+2 h(r, s)
$$

for all $p, q, r, s$ in $G$ if and only if $f, g$ and $h$ are of the form

$$
\begin{aligned}
& f(p, q)=\theta_{1}(p)+\theta_{1}(q)-\theta_{2}(p)+\theta_{2}(q)+\psi\left(p q^{-1}, p q^{-1}\right)+\beta-\alpha \\
& g(p, q)=\theta_{1}(p)+\theta_{1}(q)-\theta_{2}(p)+\theta_{2}(q)+\psi\left(p q^{-1}, p q^{-1}\right)+\beta+\alpha \\
& h(p, q)=\theta_{1}(p)+\theta_{1}(q)+\psi\left(p q^{-1}, p q^{-1}\right)+\alpha,
\end{aligned}
$$

where $\theta_{1}, \theta_{2} \in \operatorname{Hom}(G, \mathbb{C}), \psi \in \operatorname{SBihom}(G \times G, \mathbb{C})$, and $\alpha$ and $\beta$ are arbitrary constants in $\mathbb{C}$. 
Proof. Since the only if part is easy to verify, we prove only the if part of the corollary. Let $r=s=e$ in (2.26). Thus,

$$
f(p, q)+g(p, q)=2 f(p, q)+2 h(e, e) .
$$

Let $\alpha$ be in $\mathbb{C}$ such that $\alpha=h(e, e)$. Thus, the previous becomes

$$
g(p, q)=f(p, q)+2 \alpha
$$

for all $p, q \in G$. Substituting this back into (2.26) we have that

$$
f(p r, q s)+f(p s, q r)+2 \alpha=2 f(p, q)+2 h(r, s)
$$

for all $p, q, r, s \in G$. Thus, defining two functions $f_{1}, h_{1}: G \times G \rightarrow \mathbb{C}$ such that $f_{1}(p, q)=f(p, q)+\alpha$ and $h_{1}(p, q)=h(p, q)-\alpha$ for all $p, q \in G$ the previous equation reduces to the following:

$$
f_{1}(p r, q s)+f_{1}(p s, q r)=2 f_{1}(p, q)+2 h_{1}(r, s)
$$

for all $p, q, r, s \in G$. This is equation (2.20), which we have previously solved in Corollary 2.4. Hence, we have the following:

$$
\begin{aligned}
& f_{1}(p, q)=\theta_{1}(p)+\theta_{1}(q)-\theta_{2}(p)+\theta_{2}(q)+\psi\left(p q^{-1}, p q^{-1}\right)+\beta \\
& h_{1}(p, q)=\theta_{1}(p)+\theta_{1}(q)+\psi\left(p q^{-1}, p q^{-1}\right)
\end{aligned}
$$

where $\theta_{1}, \theta_{2}: G \rightarrow \mathbb{C}$ are additive homomorphisms, $\psi: G \rightarrow \mathbb{C}$ is an additive symmetric bi-homomorphism, and $\beta$ is an arbitrary constant in $\mathbb{C}$. Therefore, using the definitions of $f_{1}$ and $h_{1}$ we obtain

$$
\begin{aligned}
& f(p, q)=\theta_{1}(p)+\theta_{1}(q)-\theta_{2}(p)+\theta_{2}(q)+\psi\left(p q^{-1}, p q^{-1}\right)+\beta-\alpha \\
& h(p, q)=\theta_{1}(p)+\theta_{1}(q)+\psi\left(p q^{-1}, p q^{-1}\right)+\alpha
\end{aligned}
$$

for all $p, q, r, s \in G$. Now, by (2.27), we get the form of $g$ as

$$
g(p, q)=\theta_{1}(p)+\theta_{1}(q)-\theta_{2}(p)+\theta_{2}(q)+\psi\left(p q^{-1}, p q^{-1}\right)+\beta+\alpha
$$

for all $p, q, r, s \in G$. This completes the proof. 
The next two lemmas along with Theorem 2.3 will be essential when we make the generalization of the functional equation (2.2) involving one unknown function to four different unknown functions.

LEMMA 2.1. Let $\phi: G \rightarrow \mathbb{C}$ be an arbitrary function. Then the following two conditions are equivalent:

(1) $\phi$ is an abelian function;

(2) $\phi$ is a central function and $\phi($ prqs $)=\phi($ qrps) for all $p, q, r, s \in G$.

Proof. First we show (1) implies (2). Let $\phi$ be an abelian function. Then for all $p, r, s \in G$ we have that $\phi(p r s)=\phi(p s r)$, equivalently $\phi(p r s)=\phi(r p s)$. Let $p=e$ in the first of the previous two equations. Then $\phi(r s)=\phi(s r)$ for all $r, s \in G$ and hence $\phi$ is a central function. Consider $\phi(p r q s)$, then using the fact that $\phi$ is central and abelian, we have the following:

$\phi(p r q s)=\phi(p(r q) s)=\phi(p s(r q))=\phi((p s) r q)=\phi((p s) q r)=\phi((p s)(q r))=\phi(q r p s)$

for all $p, q, r, s \in G$. This shows that (1) implies (2).

The implication (2) implies (1) is obvious and the proof is now complete.

LEMMA 2.2. The function $f: G \times G \rightarrow \mathbb{C}$ satisfies the functional equation

$$
f(p r, q s)-f(p s, q r)=f(r, s)-f(s, r)
$$

for all $p, q, r, s$ in $G$ if and only if $f$ is of the form

$$
f(p, r)=\theta(p)-\theta(r)+\phi(p r)
$$

for all $p, r \in G$, where $\theta \in \operatorname{Hom}(G, \mathbb{C})$ and $\phi: G \rightarrow \mathbb{C}$ is an arbitrary abelian function. 
Proof. We begin by interchanging $p$ and $q$ in (2.28). Thus, we have

$$
f(q r, p s)-f(q s, p r)=f(r, s)-f(s, r) .
$$

Subtracting (2.29) from (2.28) we obtain

$$
f(p r, q s)+f(q s, p r)=f(q r, p s)+f(p s, q r)
$$

for all $p, q, r, s \in G$. Defining a function $g: G \times G \rightarrow \mathbb{C}$ such that $g(p r, q s)=$ $f(p r, q s)+f(q s, p r)$ the previous equation reduces to

$$
g(p r, q s)=g(q r, p s)
$$

for all $p, q, r, s \in G$. Letting $r=s=e$ in the previous, we find that $g$ is a symmetric function. Letting $q=s=e$ in the previous equation and using the fact that $g$ is symmetric we find that $g(p r, e)=g(r, p)=g(p, r)=g(r p, e)$ for all $p, r \in G$. Defining a function $\phi: G \rightarrow \mathbb{C}$ such that $\phi(p r)=g(p r, e)$ for all $p, r \in G$ we have that $\phi(p r)=\phi(r p)$. Thus, $\phi$ is an arbitrary central function such that $\phi($ prqs $)=\phi(q r p s)$ for all $p, q, r, s \in G$. Applying our previous lemma we have that $\phi$ is an abelian function. Since $\phi$ is an arbitrary abelian function, $2 \phi$ is also an arbitrary abelian function. Thus, by the way $g$ was defined

$$
2 \phi(p r)=f(p, r)+f(r, p)
$$

for all $p, r \in G$. Now, adding (2.29) and (2.28) we get the following:

$$
f(p r, q s)-f(p s, q r)+f(q r, p s)-f(q s, p r)=2 f(r, s)-2 f(s, r)
$$

for all $p, r, q, s \in G$. Defining a function $h: G \times G \rightarrow \mathbb{C}$ such that $2 h(p r, q s)=$ $f(p r, q s)-f(q s, p r)$, the previous becomes

$$
2 h(p r, q s)+2 h(q r, p s)=4 h(r, s)
$$

for all $p, q, r, s \in G$. Letting $r=s=e$ in the definition of $h$ we have that

$$
2 h(p, q)=f(p, q)-f(q, p)
$$


for all $p, q \in G$. Interchanging $p$ and $q$ in the previous and adding we find

$$
2 h(p, q)+2 h(q, p)=0 .
$$

Therefore, $h$ is an anti-symmetric function. Now, letting $q=s=e$ in (2.31) yields

$$
2 h(p r, e)+2 h(r, p)=4 h(r, e) .
$$

Define a function $k: G \times G \rightarrow \mathbb{C}$ such that $k(p)=h(p, e)$ for all $p \in G$. Thus,

$$
2 h(r, p)=4 k(r)-2 k(p r) .
$$

Switching $p$ and $r$ and adding the two equations together yields

$$
2 h(r, p)+2 h(p, r)=4 k(r)-2 k(p r)+4 k(p)-2 k(r p) .
$$

Since $h$ is an anti-symmetric function, the previous reduces to

$$
k(p r)+k(r p)=2 k(p)+2 k(r)
$$

for all $p, r \in G$. Therefore, from Theorem 2.1 we obtain $k$ is an additive homomorphism. Using the definitions of $k$ and $h$, we obtain

$$
f(p, r)-f(r, p)=4 k(p)-2 k(r p)
$$

for all $p, r \in G$. Adding the last equation to (2.30) we find that

$$
f(p, r)=k(p)-k(r)+\phi(p r)
$$

for all $p, r \in G$. Renaming $k$ as $\theta$ we have the asserted solution.

\subsection{The Soltuion of the Generalizaed Linear Functional}

We are now ready to prove the main result of this chapter. 
THEOREM 2.4. The functions $f, g, h, k: G \times G \rightarrow \mathbb{C}$ satisfy the functional equation

$$
f(p r, q s)+g(p s, q r)=h(p, q)+k(r, s)
$$

for all $p, q, r, s$ in $G$ if and only if $f, g, h$ and $k$ are of the form

$$
\begin{aligned}
& f(p, q)=\theta(p, q)+\psi\left(p q^{-1}, p q^{-1}\right)+\theta_{3}(p)-\theta_{3}(q)+\phi(p q)+\alpha+\beta \\
& g(p, q)=\theta(p, q)+\psi\left(p q^{-1}, p q^{-1}\right)-\theta_{3}(p)+\theta_{3}(q)-\phi(p q)+\alpha+\beta \\
& h(p, q)=2 \theta(p, q)+2 \psi\left(p q^{-1}, p q^{-1}\right)+2 \beta \\
& k(p, q)=2 \theta_{1}(p)+2 \theta_{1}(q)+2 \theta_{3}(p)-2 \theta_{3}(q)+2 \psi\left(p q^{-1}, p q^{-1}\right)+2 \alpha,
\end{aligned}
$$

where

$$
\theta(p, q)=\theta_{1}(p)+\theta_{1}(q)-\theta_{2}(p)+\theta_{2}(q),
$$

$\theta_{1}, \theta_{2}, \theta_{3} \in \operatorname{Hom}(G, \mathbb{C}), \psi \in \operatorname{SBihom}(G \times G, \mathbb{C}), \phi: G \rightarrow \mathbb{C}$ is an arbitrary abelian function, and $\alpha$ and $\beta$ are arbitrary constants in $\mathbb{C}$.

Proof. The only if part is easy to show. So we only prove the if part of the theorem. Let $r=s=e$ in (2.32). Thus,

$$
f(p, q)+g(p, q)=h(p, q)+k(e, e) .
$$

Let $\alpha$ be in $\mathbb{C}$ such that $\alpha=k(e, e)$. The previous becomes

$$
h(p, q)=f(p, q)+g(p, q)-\alpha
$$

for all $p, q \in G$. Now, let $p=q=e$ in (2.32). Thus,

$$
f(r, s)+g(s, r)=h(e, e)+k(r, s) .
$$

Let $\beta$ be in $\mathbb{C}$ such that $\beta=h(e, e)$. The previous becomes

$$
k(r, s)=f(r, s)+g(s, r)-\beta
$$


for all $r, s \in G$. Substituting (2.33) and (2.34) into (2.32) we have

$$
f(p r, q s)+g(p s, q r)=f(p, q)+g(p, q)+f(r, s)+g(s, r)-\alpha-\beta
$$

for all $p, q, r, s \in G$. Now, interchanging $r$ with $s$ in (2.35) and adding the resulting equation to $(2.35)$, we get

$(f+g)(p r, q s)+(f+g)(p s, q r)=2(f+g)(p, q)+(f+g)(r, s)+(f+g)(s, r)-2 \alpha-2 \beta$.

Defining a function $\ell: G \times G \rightarrow \mathbb{C}$ such that $\ell(p, q)=f(p, q)+g(p, q)-\alpha-\beta$ the previous reduces to the following:

$$
\ell(p r, q s)+\ell(p s, q r)=2 \ell(p, q)+\ell(r, s)+\ell(s, r)
$$

for all $p, q, r, s \in G$. Using Theorem 2.3 we obtain $\ell$ as

$$
\ell(p, q)=\theta_{1}(p)+\theta_{1}(q)-\theta_{2}(p)+\theta_{2}(q)+\psi\left(p q^{-1}, p q^{-1}\right)
$$

where $\theta_{1}, \theta_{2}: G \rightarrow \mathbb{C}$ are additive homomorphisms and $\psi: G \times G \rightarrow \mathbb{C}$ is an additive symmetric bi-homomorphism. Since $\theta_{1}$ and $\theta_{2}$ are additive homomorphisms we have that $2 \theta_{1}$ and $2 \theta_{2}$ are also additive homomorphisms. Similarly, $2 \psi$ is also an additive symmetric bi-homomorphism. Therefore,

$$
\ell(p, q)=2 \theta_{1}(p)+2 \theta_{1}(q)-2 \theta_{2}(p)+2 \theta_{2}(q)+2 \psi\left(p q^{-1}, p q^{-1}\right)
$$

and hence

$$
\begin{aligned}
f(p, q)+g(p, q)=2 \theta_{1}(p)+2 \theta_{1}(q) & -2 \theta_{2}(p)+2 \theta_{2}(q) \\
& +2 \psi\left(p q^{-1}, p q^{-1}\right)+\alpha+\beta
\end{aligned}
$$

for all $p, q \in G$.

Now, interchanging $s$ and $r$ in (2.35) we have the following:

$$
f(p s, q r)+g(p r, q s)=f(p, q)+g(p, q)+f(s, r)+g(r, s)-\alpha-\beta .
$$


Subtracting this from $(2.35)$ yields

$$
(f-g)(p r, q s)-(f-g)(p s, q r)=(f-g)(r, s)-(f-g)(s, r)
$$

for all $p, q, r, s \in G$. Defining a function $m: G \times G \rightarrow \mathbb{C}$ such that $m(p, q)=$ $f(p, q)-g(p, q)$ for all $p, q \in G$ the previous reduces to

$$
m(p r, q s)-m(p s, q r)=m(r, s)-m(s, r)
$$

for all $p, q, r, s \in G$. From Lemma 2.2 we know that

$$
m(p, q)=\theta_{3}(p)-\theta_{3}(q)+\phi(p q)
$$

for all $p, q \in G$ where $\theta_{3}: G \times G \rightarrow \mathbb{C}$ is an additive homomorphism and $\phi$ : $G \times G \rightarrow \mathbb{C}$ is an arbitrary abelian function. Since $\theta_{3}$ is an additive homomorphism and $\phi$ is an arbitrary abelian function we have that $2 \theta_{3}$ is also additive and $2 \phi$ is an arbitrary abelian function. Therefore,

$$
f(p, q)-g(p, q)=2 \theta_{3}(p)-2 \theta_{3}(q)+2 \phi(p q)
$$

Since $\alpha$ and $\beta$ are constants in $\mathbb{C}, \frac{1}{2} \alpha$ and $\frac{1}{2} \beta$ are also constants, say $\delta$ and $\gamma$ respectively. Now, adding (2.36) and (2.37) we have the following:

$$
\begin{aligned}
f(p, q)=\theta_{1}(p)+\theta_{1}(q)-\theta_{2}(p)+\theta_{2}(q) & +\psi\left(p q^{-1}, p q^{-1}\right) \\
& +\theta_{3}(p)-\theta_{3}(q)+\phi(p q)+\delta+\gamma
\end{aligned}
$$

for all $p, q \in G$. Now, subtracting (2.37) from (2.36) we have the following:

$$
\begin{aligned}
g(p, q)=\theta_{1}(p)+\theta_{1}(q)-\theta_{2}(p)+\theta_{2}(q) & +\psi\left(p q^{-1}, p q^{-1}\right) \\
& -\theta_{3}(p)+\theta_{3}(q)-\phi(p q)+\delta+\gamma
\end{aligned}
$$

for all $p, q \in G$. Since we have found both $f$ and $g$ we can now find $h$ and $k$. Substituting (2.36) into (2.33) yields

$$
h(p, q)=2 \theta_{1}(p)+2 \theta_{1}(q)-2 \theta_{2}(p)+2 \theta_{2}(q)+2 \psi\left(p q^{-1}, p q^{-1}\right)+2 \gamma
$$


for all $p, q \in G$. Substituting $f$ and $g$ in (2.34) and using the fact that $\psi\left(p q^{-1}, p q^{-1}\right)=$ $\psi\left(q p^{-1}, q p^{-1}\right)$ we get

$$
\begin{aligned}
k(p, q)=\theta_{1}(p)+ & \theta_{1}(q)-\theta_{2}(p)+\theta_{2}(q)+\psi\left(p q^{-1}, p q^{-1}\right) \\
& +\theta_{3}(p)-\theta_{3}(q)+\phi(p q)+\delta+\gamma \\
& +\theta_{1}(q)+\theta_{1}(p)-\theta_{2}(q)+\theta_{2}(p)+\psi\left(q p^{-1}, q p^{-1}\right) \\
& -\theta_{3}(q)+\theta_{3}(p)-\phi(q p)+\delta+\gamma-2 \gamma \\
=2 \theta_{1}(p) & +2 \theta_{1}(q)+2 \theta_{3}(p)-2 \theta_{3}(q)+2 \psi\left(p q^{-1}, p q^{-1}\right)+2 \delta
\end{aligned}
$$

for all $p, q \in G$. Hence, the proof is complete.

REMARK 2.1. The field of complex numbers has two binary operations, addition and multiplication. The previous proofs given in this chapter rely solely on the additivity of the complex numbers. The additivity alone gives us an abelian group under addition. Hence, the field of complex numbers can be replaced by the group of complex numbers under addition.

\subsection{Several Examples Related to the Linear Functional Equation}

We have shown that $f: G \times G \rightarrow \mathbb{C}$ is a solution of the functional equation

$$
f(p r, q s)+f(p s, q r)=2 f(p, q)+2 f(r, s)
$$

for all $p, q, r, s \in G$ if and only if $f$ is of the form

$$
f(p, r)=\theta(p)+\theta(r)+\psi\left(p r^{-1}, p r^{-1}\right)
$$

where $\theta \in \operatorname{Hom}(G, \mathbb{C})$ and $\psi \in \operatorname{SBihom}(G \times G, \mathbb{C})$. When $G$ is an abelian group the above functional equation can be written as follows:

$$
f(p+r, q+s)+f(p+s, q+r)=2 f(p, q)+2 f(r, s) .
$$


In this section, we will present several examples of functions that satisfy the previous functional equation. We begin with examples in which the domain is an abelian group.

Let $(\mathbb{R},+)$ denote the group of real numbers under addition and $(\mathbb{C},+)$ the group of complex numbers under addition. Let $p$ be a complex number, then $\bar{p}$ represents the complex conjugate of $p$.

EXAMPLE 2.1. Let $\theta:(\mathbb{R},+) \rightarrow(\mathbb{C},+)$ be a continuous additive function. Therefore, $\theta \in \operatorname{Hom}((\mathbb{R},+),(\mathbb{C},+))$. From $[13]$ we have that $\theta(p)=$ a p for all $p \in(\mathbb{R},+)$, where $a$ is an arbitrary complex constant.

Let $\psi:(\mathbb{R},+) \times(\mathbb{R},+) \rightarrow(\mathbb{C},+)$ be a continuous bi-additive function. Thus, from [13] we have that $\psi(p, q)=b p q$ for all $p, q \in(\mathbb{R},+)$, where $b$ is an arbitrary complex constant. By the commutativity of the real numbers we have that $\psi(p, q)=$ $b p q=b q p=\psi(q, p)$ and hence $\psi \in \operatorname{SBihom}((\mathbb{R},+) \times(\mathbb{R},+),(\mathbb{C},+))$.

Therefore,

$$
\begin{aligned}
f(p, q) & =\theta(p)+\theta(q)+\psi(p-q, p-q) \\
& =a p+a q+b(p-q)(p-q)
\end{aligned}
$$

where $a$ and $b$ are arbitrary complex constants is a solution of the given functional equation for all $p, q, r, s \in(\mathbb{R},+)$.

EXAMPLE 2.2. Let $\theta:(\mathbb{C},+) \rightarrow \mathbb{C}$ be a continuous additive function. Therefore, $\theta \in \operatorname{Hom}((\mathbb{C},+), \mathbb{C})$. It is known, from [13], that $\theta(p)=a_{1} p+a_{2} \bar{p}$ for all $p \in(\mathbb{C},+)$, where $a_{1}$ and $a_{2}$ are arbitrary complex constants.

Let $\psi:(\mathbb{C},+) \times(\mathbb{C},+) \rightarrow \mathbb{C}$ be a continuous bi-additive function. From [13] we get that $\psi(p, q)=b(p+\bar{p})(q+\bar{q})$ for all $p, q \in(\mathbb{C},+)$, where $b$ is an arbitrary complex constant. By the commutativity of the complex numbers we have that $\psi(p, q)=b(p+\bar{p})(q+\bar{q})=b(q+\bar{q})(p+\bar{p})=\psi(q, p)$ and hence $\psi \in$ $\operatorname{SBihom}((\mathbb{C},+) \times(\mathbb{C},+), \mathbb{C})$. 
It can be shown that

$$
\begin{aligned}
f(p, q) & =\theta(p)+\theta(q)+\psi(p-q, p-q) \\
& =a_{1} p+a_{2} \bar{p}+a_{1} q+a_{2} \bar{q}+b(p-q+\overline{p-q})(p-q+\overline{p-q}) \\
& =a_{1} p+a_{2} \bar{p}+a_{1} q+a_{2} \bar{q}+b(p-q+\bar{p}-\bar{q})(p-q+\bar{p}-\bar{q})
\end{aligned}
$$

where $a_{1}, a_{2}$, and $b$ are arbitrary complex constants is a solution of the given functional equation for all $p, q, r, s$.

Lastly, we will consider an example in which the domain is a non-commutative group. In this case, the functional equation with multiplictive notation is used, $f(p r, q s)+f(p s, q r)=2 f(p, q)+2 f(r, s)$.

EXAMPLE 2.3. Consider the group of two-by-two invertible matrices over the reals,

$$
G L_{2}(\mathbb{R})=\left\{\left[\begin{array}{ll}
a & b \\
c & d
\end{array}\right] \mid a, b, c, d \in \mathbb{R}, a d-b c \neq 0\right\}
$$

- Let $\theta: G \rightarrow \mathbb{C}$ be a function such that $\theta(P)=\log (|\operatorname{det}(P)|)$ for all $P \in G L_{2}(\mathbb{R})$. Then we have for all $P, Q \in G L_{2}(\mathbb{R})$

$$
\begin{aligned}
\theta(P Q) & =\log (|\operatorname{det}(P Q)|)=\log (|\operatorname{det}(P) \cdot \operatorname{det}(Q)|)=\log (|\operatorname{det}(P)| \cdot|\operatorname{det}(Q)|) \\
& =\log (|\operatorname{det}(P)|)+\log (|\operatorname{det}(Q)|)=\theta(P)+\theta(Q) .
\end{aligned}
$$

Therefore, $\theta \in \operatorname{Hom}\left(G L_{2}(\mathbb{R}), \mathbb{C}\right)$. Define $\psi: G L_{2}(\mathbb{R}) \times G L_{2}(\mathbb{R}) \rightarrow \mathbb{C}$ such that $\psi(P, Q)=\log (|\operatorname{det}(P)|) \cdot \log (|\operatorname{det}(Q)|)$ for all $P, Q \in G L_{2}(\mathbb{R})$. Thus for all 
$P, R, Q, S \in G L_{2}(\mathbb{R})$ the following holds:

$$
\begin{aligned}
\psi(P R, Q S)= & \log (|\operatorname{det}(P R)|) \cdot \log (|\operatorname{det}(Q S)|) \\
= & \log (|\operatorname{det}(P) \cdot \operatorname{det}(R)|) \cdot \log (|\operatorname{det}(Q) \cdot \operatorname{det}(S)|) \\
= & \log (|\operatorname{det}(P)| \cdot|\operatorname{det}(R)|) \cdot \log (|\operatorname{det}(Q)| \cdot|\operatorname{det}(S)|) \\
= & (\log (|\operatorname{det}(P)|)+\log (|\operatorname{det}(R)|)) \cdot(\log (|\operatorname{det}(Q)|)+\log (|\operatorname{det}(S)|)) \\
= & \log (|\operatorname{det}(P)|) \cdot \log (|\operatorname{det}(Q)|)+\log (|\operatorname{det}(P)|) \cdot \log (|\operatorname{det}(S)|) \\
& \quad+\log (|\operatorname{det}(R)|) \cdot \log (|\operatorname{det}(Q)|)+\log (|\operatorname{det}(R)|) \cdot \log (|\operatorname{det}(S)|) \\
= & \psi(P, Q)+\psi(P, S)+\psi(R, Q)+\psi(R, S) .
\end{aligned}
$$

Therefore, $\psi$ is a bi-homomorphism and

$$
\begin{aligned}
\psi(P, Q) & =\log (|\operatorname{det}(P)|) \cdot \log (|\operatorname{det}(Q)|) \\
& =\log (|\operatorname{det}(Q)|) \cdot \log (|\operatorname{det}(P)|) \\
& =\psi(Q, P)
\end{aligned}
$$

for all $P, Q \in G L_{2}(\mathbb{R})$. Hence, $\psi \in \operatorname{SBihom}\left(G L_{2}(\mathbb{R}) \times G L_{2}(\mathbb{R}), \mathbb{C}\right)$. We have that

$$
\begin{aligned}
f(P, R) & =\theta(P)+\theta(R)+\psi\left(P R^{-1}, P R^{-1}\right) \\
& =\log (|\operatorname{det}(P)|)+\log (|\operatorname{det}(R)|)+\log (|\operatorname{det}(P)|) \cdot \log (|\operatorname{det}(R)|)
\end{aligned}
$$

for all $P, R \in G L_{2}(\mathbb{R})$ is a solution of the given functional equation. 


\section{CHAPTER 3}

\section{A NON-LINEAR FUNCTIONAL EQUATION ON GROUPS ARISING FROM STOCHASTIC DISTANCE MEASURES}

\subsection{Introduction: A Non-Linear Functional Equation}

The main goal of this Chapter is to determine the structure of the general solution $f: G \times G \rightarrow \mathbb{C}$ of the non-linear functional equation

$$
f(p r, q s)+f(p s, q r)=f(p, q) f(r, s)
$$

on arbitrary groups. If $f$ is an abelian function, then the solution of (3.1) is of the

form $f(p, r)=m_{1}(p) m_{2}(r)+m_{1}(r) m_{2}(p)$, where $m_{1}$ and $m_{2}$ are two characters (not necessarily distinct) on $G$. In the case that there are no restrictions on $f$, whether or not there is a $c$ in the center of $G$ such that $2 f\left(c^{2}, e\right)=f(c, e)^{2}$ gives us a different picture for the structure of the solution. Specifically, we prove the following: if such a $c$ exists, then $f(p, r)=m_{1}(p) m_{2}(r)+m_{1}(r) m_{2}(p)$ for two distinct characters $m_{1}$, $m_{2}$ on $G$.

One should note that the work within this Chapter has been submitted for possible publication in the journal Mathematicae Debrecen [4].

\subsection{Previous Results on A Related Functional Equation}

We will need the solution of the symmetrized sine functional equation

$$
f(x y)+f(y x)=2 f(x) g(y)+2 f(y) g(x)
$$


on an arbitrary group. Recently, this equation was studied in [16] by Yang when $f$ and $g$ were defined on an arbitrary group $G$ and took values on the field of complex numbers $\mathbb{C}$. The following two theorems due to Yang (see [16]) will be very useful in proving our result. Recall that for any function $f$ we will use the following notation: $f_{x}(y)=f(x y)-f(x) f(y)$ for all $x, y \in G$.

THEOREM 3.1. Let $G$ be a group. Let $f, g: G \rightarrow \mathbb{C}$ be solutions of the functional equation (3.2) for all $x, y \in G$. Further, suppose that $g$ is abelian. Then either

(i) $g=\pi$, a character on $G$; or

(ii) $g=\frac{\phi(x)+\psi(x)}{2}$ for two distinct characters $\phi, \psi$ on $G$.

THEOREM 3.2. Let $G$ be a group. Let $f, g: G \rightarrow \mathbb{C}$ be solutions of the functional equation (3.2) for all $x, y \in G$.

(i) If $f_{c}(c)=0$ with $c \in Z(G)$, then

$$
f_{c}(x)=0 \quad \text { for all } x \in G \text {. }
$$

(ii) If there is a $c \in Z(G)$ such that $f_{c}(c) \neq 0$, then there are $0 \neq \lambda \in \mathbb{C}$ and two distinct characters $\phi, \psi: G \rightarrow \mathbb{C}^{\star}$ such that

$$
f(x)=\lambda(\phi(x)-\psi(x)) \quad \text { and } \quad g(x)=\frac{\phi(x)+\psi(x)}{2} \quad \forall x \in G .
$$

\subsection{A Structure Theorem}

In this section, we first consider the solution of (3.1) on an arbitrary group $G$ when $f$ is an abelian function. Then, using Theorem 3.2 and some ideas from [2] and [1], we determine the structure of the general solution on an arbitrary group $G$. 
THEOREM 3.3. Let $G$ be a group. Let $f: G \times G \rightarrow \mathbb{C}$ be a non-zero abelian function that is a solution of the functional equation (3.1) for all $p, q, r, s \in G$. Then

$$
f(p, r)=m_{1}(p) m_{2}(r)+m_{2}(p) m_{1}(r)
$$

for all $p, r \in G$, where $m_{1}, m_{2}: G \rightarrow \mathbb{C}^{\star}$ are characters.

Proof. Let $r=s=e$ in (3.1), we have

$$
f(p, q)(2-f(e, e))=0
$$

for all $p, q \in G$. Since $f \not \equiv 0$ we see that $f(e, e)=2$. Now, $q=s=e$ in (3.1) yields

$$
f(p r, e)+f(p, r)=f(p, e) f(r, e)
$$

for all $p, r \in G$. Define a function $g: G \rightarrow \mathbb{C}$ such that $g(x)=f(x, e)$. By definition, $f$ abelian implies $g$ is abelian and hence central. Using the definition of $g$, equation (3.4) becomes

$$
f(p, r)=g(p) g(r)-g(p r)
$$

One can see that $g$ being a central function implies $f$ is symmetric. Since $f \not \equiv 0$, we see that $g$ is not a character. Letting $s=e$ in (3.1) we find that

$$
f(p r, q)+f(p, q r)=f(p, q) g(r)
$$

for all $p, q, r \in G$. Now, using (3.5) in the last equality yields:

$$
g(p r) g(q)-g(p r q)+g(p) g(q r)-g(p q r)=(g(p) g(q)-g(p q)) g(r) .
$$

Since $g$ is abelian (3.6) becomes

$$
g(p r) g(q)-2 g(p q r)=g(p) g(q) g(r)-g(p) g(q r)-g(p q) g(r)
$$

for all $p, q, r \in G$. Fix $q \in G$ and define a function $\ell: G \rightarrow \mathbb{C}$ such that $\ell(p)=$ $g(p) g(q)-2 g(p q)$ for all $p \in G$. Using the definition of $\ell$, the previous equality now reduces to

$$
\ell(p r)=\ell(p) \frac{g(r)}{2}+\ell(r) \frac{g(p)}{2}
$$


for all $p, r \in G$. Since $g$ is abelian we have that $\ell$ is central, therefore

$$
\ell(p r)+\ell(r p)=\ell(p) g(r)+\ell(r) g(p)
$$

for all $p, r \in G$. Thus from Theorem 3.1 (also from [1] or [6]) we have that $g(p)=2 m(p)$ where $m$ is a character on $G$ or $g(p)=m_{1}(p)+m_{2}(p)$ for two distinct characters $m_{1}, m_{2}$ on $G$. If $g(p)=2 m(p)$ then by (3.5) we have that

$$
f(p, r)=2 m(p r)
$$

for all $p, r \in G$. Hence $f$ in (3.8) is of the form (3.3) with $m_{1}=m_{2}=m$. If $g(p)=m_{1}(p)+m_{2}(p)$, then substituting back into (3.5) we see that

$$
\begin{aligned}
f(p, r) & =\left(m_{1}(p)+m_{2}(p)\right)\left(m_{1}(r)+m_{2}(r)\right)-\left(m_{1}(p r)+m_{2}(p r)\right) \\
& =m_{1}(p) m_{2}(r)+m_{2}(p) m_{1}(r)
\end{aligned}
$$

for all $p, r \in G$, which gives us (3.3).

Now, we will consider when there are no restrictions on the function $f$.

THEOREM 3.4. Let $G$ be a group and e be the identity element of $G$. Let $f: G \times$ $G \rightarrow \mathbb{C}$ be a non-zero solution of the functional equation (3.1) for all $p, q, r, s \in G$, then one of the following holds:

(i) if there is a $c \in Z(G)$ such that $2 f\left(c^{2}, e\right) \neq f(c, e)^{2}$, then there are two distinct characters $m_{1}, m_{2}$ on $G$ such that

$$
f(p, r)=m_{1}(p) m_{2}(r)+m_{2}(p) m_{1}(r),
$$

for all $p, r \in G$;

(ii) if $2 f\left(c^{2}, e\right)=f(c, e)^{2}$ with $c \in Z(G)$, then

$$
2 f(c, x)=f(c, e) f(x, e)
$$

for all $x \in G$. 
Proof. Let $r=s=e$ in (3.1), we have

$$
f(p, q)(2-f(e, e))=0
$$

for all $p, q \in G$. Since $f \not \equiv 0$ we see that $f(e, e)=2$. Let $p=q=e$ in (3.1), then using the fact that $f(e, e)=2$ we get the following for all $r, s \in G$

$$
f(r, s)+f(s, r)=f(e, e) f(r, s)=2 f(r, s) .
$$

Therefore, $f$ is symmetric. Now, $q=s=e$ in (3.1) yields

$$
f(p r, e)+f(p, r)=f(p, e) f(r, e)
$$

for all $p, r \in G$. Define a function $g: G \rightarrow \mathbb{C}$ such that $g(x)=f(x, e)$. Therefore, $g(e)=2$ and the equation (3.11) becomes

$$
f(p, r)=g(p) g(r)-g(p r)
$$

One can see that since $f$ is symmetric, $g$ is a central function. Since $f \not \equiv 0$, we see that $g$ is not a character. Letting $s=e$ in (3.1) we find that

$$
f(p r, q)+f(p, q r)=f(p, q) g(r)
$$

for all $p, q, r \in G$. Now, using (3.12) in the last equality yields:

$$
\begin{aligned}
g(p r) g(q)-g(p r q)+g(p) g(q r)-g(p q r) & =(g(p) g(q)-g(p q)) g(r) \\
g(p) g(q r)-g(p r q)-g(p q r) & =g(p) g(q) g(r)-g(p q) g(r)-g(p r) g(q) .
\end{aligned}
$$

Multiplying the last equation by 2 and rewriting we find that

$$
\begin{aligned}
g(p) g(q r) & -2 g(p q r)+g(p) g(q r)-2 g(p r q) \\
& =(g(p) g(q)-2 g(p q)) g(r)+(g(p) g(r)-2 g(p r)) g(q)
\end{aligned}
$$

for all $p, q, r \in G$. Now, fix $p \in G$ and define a function $h: G \rightarrow \mathbb{C}$ as follows:

$$
h(r)=g(p) g(r)-2 g(p r)
$$


for all $r \in G$. Hence by (3.14) and the fact that $g$ is central, the equation (3.13) reduces to

$$
h(q r)+h(r q)=2 h(q) \frac{g(r)}{2}+2 h(r) \frac{g(q)}{2} .
$$

Defining a function $k: G \rightarrow \mathbb{C}$ such that $k(r)=\frac{g(r)}{2}$ for all $r \in G$, the previous equation becomes

$$
h(q r)+h(r q)=2 h(q) k(r)+2 h(r) k(q)
$$

for all $q, r \in G$, which is equation (3.2). Thus, we can apply Theorem 3.2. Using the definitions of $h$ and $k$ we have that for $c \in Z(G)$

$$
k_{c}(c)=k\left(c^{2}\right)-k(c)^{2}=\frac{g\left(c^{2}\right)}{2}-\left(\frac{g(c)}{2}\right)^{2}=\frac{f\left(c^{2}, e\right)}{2}-\frac{f(c, e)^{2}}{4} .
$$

Hence, $k_{c}(c)=0$ if and only if

$$
2 f\left(c^{2}, e\right)=f(c, e)^{2}
$$

for some $c \in Z(G)$.

If there is a $c \in Z(G)$ such that $2 f\left(c^{2}, e\right) \neq f(c, e)^{2}$ (that is $k_{c}(c) \neq 0$ ), then there are two distinct characters $m_{1}, m_{2}: G \rightarrow \mathbb{C}^{\star}$ and $0 \neq \lambda \in \mathbb{C}$ such that

$$
k(x)=\frac{m_{1}(x)+m_{2}(x)}{2} \text { and } \quad h(x)=\lambda\left(m_{1}(x)-m_{2}(x)\right)
$$

for all $x \in G$. Hence, by the definitions of $k$ and $g$ we have the following:

$$
g(x)=m_{1}(x)+m_{2}(x)
$$

for all $x \in G$. Now, using the previous equation in (3.12) yields

$$
\begin{aligned}
f(p, r) & =\left(m_{1}(p)+m_{2}(p)\right)\left(m_{1}(r)+m_{2}(r)\right)-\left(m_{1}(p r)+m_{2}(p r)\right) \\
& =m_{1}(p) m_{2}(r)+m_{2}(p) m_{1}(r)
\end{aligned}
$$

for all $p, r, G$, which is (3.9) and thus case (i) is complete. 
From Theorem 3.2 we know that if $k_{c}(c)=0$ with $c \in Z(G)$, then $k_{c}=0$. Since $k_{c}=0$, i.e. $k_{c}(x)=0$ for all $x \in G$ and $c \in Z(G)$, we have the following:

$$
k(c x)-k(c) k(x)=0,
$$

which implies

$$
2 g(c x)=g(c) g(x)
$$

and from the definition of $g$

$$
2 f(c x, e)=f(c, e) f(x, e) .
$$

Using (3.12) and (3.16) we have the following:

$$
f(x, c)=g(x) g(c)-g(x c)=g(x c)=f(x c, e)
$$

for all $x \in G$ and $c \in Z(G)$. Now, using (3.17), the fact that $c \in Z(G)$, and the previous equality we get that

$$
2 f(c, x)=f(c, e) f(x, e)
$$

for all $x \in G$ and $c \in Z(G)$, which is (3.10). Hence, case (ii) of the theorem is now complete. This finishes the proof of the theorem.

REMARK 3.1. If $Z(G)=\{e\}$, then $c=e$. A group in which $Z(G)=\{e\}$ is refered to as a centerless group. Since $f(e, e)=2,(3.15)$ and (3.18) hold. Therefore, in the case that the group $G$ is centerless Theorem 3.4 provides no information in regards to the solution of (3.1) on such a group. For instance, consider the alternating group $A_{5}$; which is the subgroup of $S_{5}$ containing the identity element and all of the even permutations of $S_{5}$. It is known that $Z\left(A_{5}\right)=\{e\}$. Therefore, the above theorem tells us nothing in regards to the solution of (3.1) on $A_{5}$. 


\section{CHAPTER 4 \\ SEVERAL GENERALIZATIONS OF A NON-LINEAR FUNCTIONAL EQUATION ON GROUPS ARISING FROM STOCHASTIC DISTANCE MEASURES}

\subsection{Introduction: Generalizations of A Non-Linear Functional Equation}

In this Chapter we consider several generalizations of the non-linear functional equation $f(p r, q s)+f(p s, q r)=f(p, q) f(r, s)$. Specifically, we determine the general structure of the solution $f, g, h: G \times G \rightarrow \mathbb{C}$ of the non-linear functional equation $f(p r, q s)+f(p s, q r)=g(p, q) h(r, s)$ for all $p, q, r, s \in G$. The structure of the solution is dependent upon how the function $h$ acts on the center of the group. In order to determine the structure of this solution, we must consider the structure of the solution of the functional equation $f(p r, q s)+f(p s, q r)=f(p, q) g(r, s)$ for all $p, q, r, s \in G$.

\subsection{Generalization Theorems}

We will now present the solution to several generalization of the functional equation $f(p r, q s)+f(p s, q r)=f(p, q) f(r, s)$. We begin with a case in which we have two functions $f$ and $g$.

THEOREM 4.1. Let $G$ be a group and e be the identity element of $G$. Let $f, g$ : $G \times G \rightarrow \mathbb{C}$ be a non-zero solution of the functional equation

$$
f(p r, q s)+f(p s, q r)=f(p, q) g(r, s)
$$


for all $p, q, r, s \in G$, then one of the following holds:

i) if there exists $c \in Z(G)$ such that $g\left(c^{2}, e\right)=g(c, e)^{2}$, then

$$
g(c x, e)=0
$$

for all $x \in G$;

ii) if there exists $c \in Z(G)$ such that $g\left(c^{2}, e\right) \neq g(c, e)^{2}$, then there exists two distinct characters $m_{1}, m_{2}: G \rightarrow \mathbb{C}^{\star}$ and $0 \neq \lambda \in \mathbb{C}$ such that

$$
\begin{aligned}
& f(p, r)=\left(\frac{\lambda+\alpha}{2}\right) m_{1}(r) m_{2}(p)+\left(\frac{\alpha-\lambda}{2}\right) m_{2}(r) m_{1}(p), \\
& g(p, r)=m_{1}(p) m_{2}(r)+m_{1}(r) m_{2}(p)
\end{aligned}
$$

for all $p, r \in G$, where $\alpha$ is an arbitrary constant in $\mathbb{C}$.

Proof. Switch $r$ and $s$ in (4.1). Then

$$
f(p s, q r)+f(p r, q s)=f(p, q) g(s, r)
$$

for all $p, q, r, s \in G$. Subtracting this from (4.1) we get that

$$
0=f(p, q)(g(r, s)-g(s, r)) .
$$

Since $f$ is not identically zero, we can conclude that $g$ is a symmetric function. Now, let $s=q=e$ in (4.1). This yields

$$
f(p r, e)+f(p, r)=f(p, e) g(r, e)
$$

for all $p, r \in G$.

Now, define a function $f_{1}: G \rightarrow \mathbb{C}$ such that $f_{1}(p)=f(p, e)$ for all $p \in G$ and a function $h: G \rightarrow \mathbb{C}$ such that $h(p)=g(p, e)=g(e, p)$ for all $p \in G$. Letting $r=s=e$ in (4.1) gives us

$$
f(p, q)(2-g(e, e))=0
$$


for all $p, q \in G$. Since $f$ is not identically, $h(e)=g(e, e)=2$. Using the definition of $f_{1}$ and the definition of $h$, we have that equation (4.2) reduces to the following:

$$
f(p, r)=f_{1}(p) h(r)-f_{1}(p r)
$$

for all $p, r \in G$.

Let $p=r=e$ in (4.1), then we get that

$$
f(e, q s)+f(s, q)=f(e, q) g(s, e)
$$

for all $q, s \in G$. Defining a function $f_{2}: G \rightarrow \mathbb{C}$ such that $f_{2}(p)=f(e, p)$ for all $p \in G$ and using the definition of $h$ the previous becomes

$$
f(s, q)=f_{2}(q) h(s)-f_{2}(q s)
$$

for all $q, s \in G$. If we consider $s=p$ and $q=r$ we get

$$
f(p, r)=f_{2}(r) h(p)-f_{2}(r p)
$$

Comparing (4.3) and (4.4) we get the following:

$$
f_{1}(p) h(r)-f_{1}(p r)=f_{2}(r) h(p)-f_{2}(r p) .
$$

This equation can be rewritten as

$$
f_{2}(r p)-f_{1}(p r)=f_{2}(r) h(p)-f_{1}(p) h(r)
$$

for all $p, r \in G$. Switching $p$ and $r$ in (4.5) yields

$$
f_{2}(p r)-f_{1}(r p)=f_{2}(p) h(r)-f_{1}(r) h(p) .
$$

Subtracting (4.6) from (4.5) and simplifying gives us the following:

$$
\left(f_{2}(r p)+f_{1}(r p)\right)-\left(f_{2}(p r)+f_{1}(p r)\right)=h(p)\left(f_{2}(r)+f_{1}(r)\right)-h(r)\left(f_{2}(p)+f_{1}(p)\right)
$$


for all $p, r \in G$. Defining a function $k_{1}: G \rightarrow \mathbb{C}$ such that $k_{1}(p)=f_{2}(p)+f_{1}(p)$ for all $p \in G$ the previous reduces to

$$
k_{1}(r p)-k_{1}(p r)=h(p) k_{1}(r)-h(r) k_{1}(p)
$$

for all $p, r \in G$.

Define $f(e, e)=\alpha$ where $\alpha \in \mathbb{C}$. Then by definition, $f(e, e)=f_{1}(e)=$ $f_{2}(e)=\alpha$. Therefore, $k_{1}(e)=2 f_{1}(e)=2 f_{2}(e)=2 \alpha$. Let $r=e$ in the previous equation (4.9). Then we have

$$
0=h(e) k_{1}(r)-h(r) k_{1}(e)
$$

which is

$$
0=2 k_{1}(r)-h(r) 2 \alpha
$$

for all $r \in G$. From the last inequality, we see that

$$
k_{1}(r)=\alpha h(r)
$$

for all $r \in G$. We will consider two cases, $\alpha=0$ and $\alpha \neq 0$.

We begin with the case when $\alpha=0$. Since $k_{1}(p)=\alpha h(p)$ for all $p \in G$, we have that $k_{1}=0$. This implies that

$$
f_{2}(p)=-f_{1}(p)
$$

for all $p \in G$. Substituting for $f_{1}$ in $(4.5)$ gives us

$$
f_{2}(r p)+f_{2}(p r)=f_{2}(r) h(p)+f_{2}(p) h(r)
$$

for all $p, r \in G$. This is the symmetrized sine equation. The solution of this equation was obtained by Yang in [16] and is stated in Theorem 3.2. From Theorem 3.2 we have two cases. If $h_{c}(c)=0$ for $c \in Z(G)$, that is, if $h\left(c^{2}\right)=h(c)^{2}$ which is $g\left(c^{2}, e\right)=g(c, e)^{2}$, then $h_{c}(x)=g(c x, e)=0$ for all $x \in G$ and $c \in Z(G)$. 
If there exists $c \in Z(G)$ such that $g\left(c^{2}, e\right) \neq g(c, e)^{2}$, then there exists a non-zero $\lambda \in \mathbb{C}$ and two distinct characters, $m_{1}, m_{2}: G \rightarrow \mathbb{C}^{\star}$ such that

$$
h(p)=m_{1}(p)+m_{2}(p) \text { and } f_{2}(p)=\lambda\left(m_{1}(p)-m_{2}(p)\right)
$$

for all $p \in G$. Using (4.4) we get

$$
f(p, r)=\lambda\left(m_{1}(r)-m_{2}(r)\right)\left(m_{1}(p)+m_{2}(p)\right)-\lambda\left(m_{1}(r p)-m_{2}(r p)\right) .
$$

Using the fact that $m_{1}$ and $m_{2}$ are characters on $G, f(p, r)$ can be rewritten as

$$
\begin{aligned}
f(p, r)=\lambda & \left(m_{1}(r) m_{1}(p)+m_{1}(r) m_{2}(p)-m_{2}(r) m_{1}(p)-m_{2}(r) m_{2}(p)\right) \\
& -\lambda\left(m_{1}(r) m_{1}(p)-m_{2}(r) m_{2}(p)\right)
\end{aligned}
$$

which simplifies to

$$
f(p, r)=\lambda\left(m_{1}(r) m_{2}(p)-m_{2}(r) m_{1}(p)\right)
$$

for all $p, r \in G$. Substituting back into (4.1) yields

$$
\begin{array}{r}
\lambda\left(m_{1}(q s) m_{2}(p r)-m_{2}(q s) m_{1}(p r)\right)+\lambda\left(m_{1}(q r) m_{2}(p s)-m_{2}(q r) m_{1}(p s)\right) \\
=\lambda\left(m_{1}(q) m_{2}(p)-m_{2}(q) m_{1}(p)\right) g(r, s) .
\end{array}
$$

Using the fact that $m_{1}$ and $m_{2}$ are characters on $G$, we can rewrite the previous as follows:

$$
\begin{array}{r}
m_{1}(q) m_{1}(s) m_{2}(p) m_{2}(r)-m_{2}(q) m_{2}(s) m_{1}(p) m_{1}(r) \\
+m_{1}(q) m_{1}(r) m_{2}(p) m_{2}(s)-m_{2}(q) m_{2}(r) m_{1}(p) m_{1}(s) \\
=\left(m_{1}(q) m_{2}(p)-m_{2}(q) m_{1}(p)\right) g(r, s) .
\end{array}
$$

The previous factors into the following:

$$
\begin{aligned}
\left(m_{1}(q) m_{2}(p)-m_{2}(q) m_{1}(p)\right) & \left(m_{1}(s) m_{2}(r)+m_{2}(s) m_{1}(r)\right) \\
= & \left(m_{1}(q) m_{2}(p)-m_{2}(q) m_{1}(p)\right) g(r, s)
\end{aligned}
$$


and thus simplifying gives us

$$
g(r, s)=m_{1}(s) m_{2}(r)+m_{2}(s) m_{1}(r)
$$

for all $p, q, r, s \in G$. Since $\lambda$ is an arbitrary non-zero constant in $\mathbb{C}$ we have that

$$
\begin{aligned}
& f(p, r)=\frac{\lambda}{2} m_{1}(r) m_{2}(p)-\frac{\lambda}{2} m_{2}(r) m_{1}(p) \\
& g(p, r)=m_{1}(r) m_{2}(p)+m_{2}(r) m_{1}(p)
\end{aligned}
$$

for all $p, r \in G$. This completes the case when $\alpha=0$.

Now, we consider the case when $\alpha \neq 0$. Adding (4.6) and (4.5) gives us

$$
f_{2}(r p)-f_{1}(p r)+f_{2}(p r)-f_{1}(r p)=f_{2}(r) h(p)-f_{1}(p) h(r)+f_{2}(p) h(r)-f_{1}(r) h(p),
$$

and hence

$$
\left(f_{2}(r p)-f_{1}(r p)\right)+\left(f_{2}(p r)-f_{1}(p r)\right)=h(p)\left(f_{2}(r)-f_{1}(r)\right)+h(r)\left(f_{2}(p)-f_{1}(p)\right)
$$

for all $p, r \in G$. Defining a function $k_{2}: G \rightarrow \mathbb{C}$ such that $k_{2}(p)=f_{2}(p)-f_{1}(p)$ for all $p \in G$ the previous reduces to

$$
k_{2}(r p)+k_{2}(p r)=h(p) k_{2}(r)+h(r) k_{2}(p)
$$

for all $p, r \in G$. This is the symmetrized sine equation. From Yang's Theorem 3.2, we have two cases. If $h_{c}(c)=0$ for $c \in Z(G)$, that is, if $h\left(c^{2}\right)=h(c)^{2}$ which is $g\left(c^{2}, e\right)=g(c, e)^{2}$ then $h_{c}(x)=g(c x, e)=0$ for all $x \in G$ and $c \in Z(G)$. This is the same condition we found when $\alpha=0$. Hence, if there exists $c \in Z(G)$ such that $g\left(c^{2}, e\right)=g(c, e)^{2}$ then $g(c x, e)=0$. This completes the first case.

If there exists $c \in Z(G)$ such that $g\left(c^{2}, e\right) \neq g(c, e)^{2}$, then there exists a non-zero $\lambda \in \mathbb{C}$ and two distinct characters, $m_{1}, m_{2}: G \rightarrow \mathbb{C}^{\star}$ such that

$$
h(p)=m_{1}(p)+m_{2}(p) \text { and } k_{2}(p)=\lambda\left(m_{1}(p)-m_{2}(p)\right)
$$


for all $p \in G$. Recall that since $\alpha \neq 0$ we have $k_{1}(r)=\alpha h(r)$. Thus, by the way $k_{2}$ and $k_{1}$ were defined in terms of $h$ we have that

$$
\begin{aligned}
& f_{2}(p)-f_{1}(p)=\lambda\left(m_{1}(p)-m_{2}(p)\right) \\
& f_{2}(p)+f_{1}(p)=\alpha\left(m_{1}(p)+m_{2}(p)\right)
\end{aligned}
$$

for all $p \in G$. From (4.3) and (4.4) we know that in order to find $f$ we need either $f_{1}$ or $f_{2}$. Thus, adding (4.9) and (4.10) and then dividing by 2 we get the following:

$$
f_{2}(p)=\left(\frac{\lambda+\alpha}{2}\right) m_{1}(p)+\left(\frac{\alpha-\lambda}{2}\right) m_{2}(p)
$$

for all $p \in G$. Now, substituting into (4.4) for both $f_{2}$ and $h$ gives us

$$
\begin{aligned}
f(p, r)= & \left.\left(\frac{\lambda+\alpha}{2}\right) m_{1}(r)+\left(\frac{\alpha-\lambda}{2}\right) m_{2}(r)\right)\left(m_{1}(p)+m_{2}(p)\right) \\
& -\left(\frac{\lambda+\alpha}{2}\right) m_{1}(r p)+\left(\frac{\alpha-\lambda}{2}\right) m_{2}(r p) .
\end{aligned}
$$

After multiplying out and then simplifying, the previous reduces to

$$
f(p, r)=\left(\frac{\lambda+\alpha}{2}\right) m_{1}(r) m_{2}(p)+\left(\frac{\alpha-\lambda}{2}\right) m_{2}(r) m_{1}(p)
$$

for all $p, r \in G$.

We can now use $f$ to find $g$. Let $p=q=e$ in (4.1). Then, since $f(e, e)=$ $\alpha \neq 0$ we get that

$$
\frac{1}{\alpha} f(r, s)+\frac{1}{\alpha} f(s, r)=g(r, s)
$$

for all $r, s \in G$. Substituting in for $f$ and then simplifying yields

$$
\begin{aligned}
g(r, s)= & \frac{1}{\alpha}\left(\left(\frac{\lambda+\alpha}{2}\right) m_{1}(r) m_{2}(s)+\left(\frac{\alpha-\lambda}{2}\right) m_{2}(r) m_{1}(s)\right) \\
& +\frac{1}{\alpha}\left(\left(\frac{\lambda+\alpha}{2}\right) m_{1}(s) m_{2}(r)+\left(\frac{\alpha-\lambda}{2}\right) m_{2}(s) m_{1}(r)\right) \\
g(r, s)= & m_{1}(r) m_{2}(s)+m_{1}(s) m_{2}(r)
\end{aligned}
$$

for all $r, s \in G$. This completes the case when $\alpha \neq 0$. One can easily see that when $g\left(c^{2}, e\right) \neq g(c, e)^{2}$ we have that $g$ takes the same form regardless of whether or not 
$\alpha$ is non-zero. It can also be seen that (4.11) is equivalent to (4.8) when $\alpha=0$. Hence, this completes the second case and the proof.

COROLLARY 4.1. Let $G$ be a group and $e$ be the identity element of $G$. Let $f, g, h: G \times G \rightarrow \mathbb{C}$ be a non-zero solution of the functional equation

$$
f(p r, q s)+f(p s, q r)=g(p, q) h(r, s)
$$

for all $p, r, q, s \in G$, then one of the following holds:

i) if there exists $c \in Z(G)$ such that $h\left(c^{2}, e\right)=\frac{2}{\beta} h(c, e)^{2}$ where $0 \neq \beta \in \mathbb{C}$, then

$$
h(c x, e)=0
$$

for all $x \in G$;

ii) if there exists $c \in Z(G)$ such that $h\left(c^{2}, e\right) \neq \frac{2}{\beta} h(c, e)^{2}$ where $0 \neq \beta \in \mathbb{C}$, then there exists two distinct characters $m_{1}, m_{2}: G \rightarrow \mathbb{C}^{\star}$ and $0 \neq \lambda \in \mathbb{C}$ such that

$$
\begin{aligned}
& f(p, r)=\left(\frac{\lambda+\alpha}{2}\right) m_{1}(r) m_{2}(p)+\left(\frac{\alpha-\lambda}{2}\right) m_{2}(r) m_{1}(p), \\
& g(p, r)=\left(\frac{\lambda+\alpha}{\beta}\right) m_{1}(r) m_{2}(p)+\left(\frac{\alpha-\lambda}{\beta}\right) m_{2}(r) m_{1}(p), \\
& h(p, r)=\frac{\beta}{2}\left(m_{1}(p) m_{2}(r)+m_{1}(r) m_{2}(p)\right)
\end{aligned}
$$

for all $p, r \in G$ where $\alpha$ is an arbitrary constant in $\mathbb{C}$.

Proof. Let $r=s=e$ in (4.12). Then

$$
2 f(p, q)=g(p, q) h(e, e)
$$

for all $p, q \in G$. If $h(e, e)=0$ then $2 f(p, q)=0$, which contradicts the assumption that $f$ is non-trivial. Therefore, $h(e, e)=\beta \neq 0$ where $\beta \in \mathbb{C}$. This gives us that

$$
\frac{2}{\beta} f(p, q)=g(p, q)
$$


for all $p, q \in G$. By substituting into (4.12) we get the following:

$$
f(p r, q s)+f(p s, q r)=\frac{2}{\beta} f(p, q) h(r, s)
$$

for all $p, q, r, s \in G$.

Defining a function $k: G \times G \rightarrow \mathbb{C}$ such that $k(p, q)=\frac{2}{\beta} h(p, q)$ the previous becomes

$$
f(p r, q s)+f(p s, q r)=f(p, q) k(r, s)
$$

for all $p, q, r, s \in G$. From Theorem 4.1 we have that if $k\left(c^{2}, e\right)=k(c, e)^{2}$ for $c \in Z(G)$, then

$$
k(c x, e)=0
$$

for all $x \in G$ and $c \in Z(G)$. Using the definition of $k$, if $\frac{2}{\beta} h\left(c^{2}, e\right)=\left(\frac{2}{\beta} h(c, e)\right)^{2}$, that is $h\left(c^{2}, e\right)=\frac{2}{\beta} h(c, e)^{2}$ for $c \in Z(G)$, then $\frac{2}{\beta} h(c x, e)=0$ and hence $h(c x, e)=0$ for all $x \in G$ and $c \in Z(G)$. This completes the first case.

If there exists $c \in Z(G)$ such that $k\left(c^{2}, e\right) \neq k(c, e)^{2}$, that is $h\left(c^{2}, e\right) \neq$ $\frac{2}{\beta} h(c, e)^{2}$, then there exists two distinct characters $m_{1}, m_{2}: G \rightarrow \mathbb{C}^{\star}$ and $\lambda \in \mathbb{C}$ that is non-zero such that

$$
\begin{aligned}
& f(p, r)=\left(\frac{\lambda+\alpha}{2}\right) m_{1}(r) m_{2}(p)+\left(\frac{\alpha-\lambda}{2}\right) m_{2}(r) m_{1}(p) \\
& k(p, r)=m_{1}(p) m_{2}(r)+m_{1}(r) m_{2}(p)
\end{aligned}
$$

for all $p, r \in G$, where $\alpha$ is an arbitrary constant in $\mathbb{C}$. From the way that $g$ in terms of $f$, substituting and simplifying yields

$$
g(p, r)=\left(\frac{\lambda+\alpha}{\beta}\right) m_{1}(r) m_{2}(p)+\left(\frac{\alpha-\lambda}{\beta}\right) m_{2}(r) m_{1}(p)
$$

for all $p, r \in G$. The definition of $k$ gives us

$$
h(p, r)=\frac{\beta}{2}\left(m_{1}(p) m_{2}(r)+m_{1}(r) m_{2}(p)\right)
$$

for all $p, r \in G$, which completes the proof. 
Now, we will consider the functional equation

$$
f(p r, q s)+f(p s, q r)=g(p, q) f(r, s)
$$

for all $p, q, r, s \in G$. If we were to take $h$ to be $f$ in Corollary 4.1 yields $\lambda=0$, which is a contradiction to our assumption in Corollary 4.1 that $\lambda \in \mathbb{C}$ is non-zero. Therefore, we consider another method.

COROLlARY 4.2. Let $G$ be a group and $e$ be the identity element of $G$. Let $f, g: G \times G \rightarrow \mathbb{C}$ be a non-zero solution of the functional equation (4.13) for all $p, q, r, s \in G$, then one of the following holds:

i) if there exists $c \in Z(G)$ such that $f\left(c^{2}, e\right)=\frac{1}{\alpha} f(c, e)^{2}$ where $0 \neq \alpha \in \mathbb{C}$ then

$$
f(c, x)=\frac{1}{\alpha} f(c, e) f(x, e)
$$

for all $x \in G$;

ii) if there exists $c \in Z(G)$ such that $f\left(c^{2}, e\right) \neq \frac{1}{\alpha} f(c, e)^{2}$ where $0 \neq \alpha \in \mathbb{C}$, then there exists two distinct characters $m_{1}, m_{2}: G \rightarrow \mathbb{C}^{\star}$ such that

$$
\begin{aligned}
& f(p, r)=\frac{\alpha}{2}\left(m_{1}(p) m_{2}(r)+m_{2}(p) m_{1}(r)\right), \\
& g(p, r)=m_{1}(p) m_{2}(r)+m_{1}(r) m_{2}(p)
\end{aligned}
$$

for all $p, r \in G$.

Proof. Let $r=s=e$ in (4.13). Then

$$
2 f(p, q)=g(p, q) f(e, e)
$$

for all $p, q \in G$. If $f(e, e)=0$ then $f(p, q)=0$ for all $p, q \in G$, which contradicts the assumption that $f$ is non-trivial. Therefore, $f(e, e)=\alpha \neq 0$ where $\alpha \in \mathbb{C}$. This gives us that

$$
\frac{2}{\alpha} f(p, q)=g(p, q)
$$


for all $p, q \in G$. By substituting into (4.13) we get the following:

$$
f(p r, q s)+f(p s, q r)=\frac{2}{\alpha} f(p, q) f(r, s)
$$

for all $p, q, r, s \in G$. Multiplying through by $\frac{2}{\alpha}$ and defining a function $h: G \times G \rightarrow \mathbb{C}$ such that $h(p, q)=\frac{2}{\alpha} f(p, q)$ the previous becomes

$$
h(p r, q s)+h(p s, q r)=h(p, q) h(r, s)
$$

for all $p, q, r, s \in G$. Therefore, from Theorem 2.2 we have that if there exists a $c \in Z(G)$ such that $2 h\left(c^{2}, e\right)=h(c, e)^{2}$, then

$$
2 h(c, x)=h(c, e) h(x, e)
$$

for all $x \in G$ and $c \in Z(G)$. From the definition of $h$ we get that if $\frac{2}{\alpha} 2 f\left(c^{2}, e\right)=$ $\left(\frac{2}{\alpha} f(c, e)\right)^{2}$, that is if $f\left(c^{2}, e\right)=\frac{1}{\alpha} f(c, e)^{2}$ for $c \in Z(G)$, then $f(c, x)=\frac{1}{\alpha} f(c, e) f(x, e)$ for all $x \in G$ and $c \in Z(G)$. This completes the first case.

If there is a $c \in Z(G)$ such that $2 h\left(c^{2}, e\right) \neq h(c, e)^{2}$, then there exists two distinct characters $m_{1}, m_{2}$ on $G$ such that

$$
h(p, r)=m_{1}(p) m_{2}(r)+m_{2}(p) m_{1}(r)
$$

for all $p, r \in G$. Therefore, if $f\left(c^{2}, e\right) \neq \frac{1}{\alpha} f(c, e)^{2}$ for $c \in Z(G)$ then

$$
f(p, r)=\frac{\alpha}{2}\left(m_{1}(p) m_{2}(r)+m_{2}(p) m_{1}(r)\right)
$$

for all $p, r \in G$. Substituting into the definition of $g$ in terms of $f$ we get that

$$
g(p, r)=m_{1}(p) m_{2}(r)+m_{2}(p) m_{1}(r)
$$

for all $p, r \in G$, which completes the second case and hence completes the proof.

Lastly, we will consider the functional equation

$$
f(p r, q s)+f(p s, q r)=g(p, q) g(r, s)
$$


for all $p, q, r, s \in G$. Taking $h$ to be $g$ in Corollary 4.1 yields $\frac{\lambda+\alpha}{\beta}=\frac{\beta}{2}$ and $\frac{\alpha-\lambda}{\beta}=\frac{\beta}{2}$. By cross multiplying and setting the two equal one can easily see that $\lambda=-\lambda$, which implies that $\lambda=0$. This is a contradiction to our assumption in Corollary 4.1 that $\lambda \in \mathbb{C}$ is non-zero. Therefore, we again must consider another method.

COROLLARY 4.3. Let $G$ be a group and $e$ be the identity element of $G$. Let $f, g: G \times G \rightarrow \mathbb{C}$ be a non-zero solution of the functional equation

$$
f(p r, q s)+f(p s, q r)=g(p, q) g(r, s)
$$

for all $p, q, r, s \in G$, then one of the following holds:

(i) if there exists $c \in Z(G)$ such that $g\left(c^{2}, e\right)=\frac{1}{\alpha} g(c, e)^{2}$ where $0 \neq \alpha \in \mathbb{C}$, then

$$
g(c, x)=\frac{1}{\alpha} g(c, e) g(x, e)
$$

for all $x \in G$;

(ii) if there exists $c \in Z(G)$ such that $g\left(c^{2}, e\right) \neq \frac{1}{\alpha} g(c, e)^{2}$ where $0 \neq \alpha \in \mathbb{C}$, then there are two distinct characters $m_{1}, m_{2}$ on $G$ such that

$$
\begin{aligned}
& f(p, r)=\frac{\alpha^{2}}{4}\left(m_{1}(p) m_{2}(r)+m_{2}(p) m_{1}(r)\right) \\
& g(p, r)=\frac{\alpha}{2}\left(m_{1}(p) m_{2}(r)+m_{2}(p) m_{1}(r)\right)
\end{aligned}
$$

for all $p, r \in G$.

Proof. Let $r=s=e$ in (4.14). Then

$$
2 f(p, q)=g(p, q) g(e, e)
$$

for all $p, q \in G$. Define $g(e, e)=\alpha$, where $\alpha$ is a complex constant. If $\alpha=0$ we get $f(p, q)=0$ for all $p, q \in G$, which contradicts our assumption that $f$ is a non-zero function. Thus, $\alpha$ is a non-zero complex constant and

$$
f(p, q)=\frac{\alpha}{2} g(p, q)
$$


for all $p, q \in G$. Substituting into (4.14) for $f$ yields the following:

$$
\frac{\alpha}{2} g(p r, q s)+\frac{\alpha}{2} g(p s, q r)=g(p, q) g(r, s)
$$

for all $p, q, r, s \in G$. Define a new function $h: G \times G \rightarrow \mathbb{C}$ such that $h(p, q)=$ $\frac{2}{\alpha} g(p, q)$. Then $g(p, q)=\frac{\alpha}{2} h(p, q)$. Using the definition of $h$, the previous becomes

$$
\frac{\alpha}{2}\left(\frac{\alpha}{2} h(p r, q s)\right)+\frac{\alpha}{2}\left(\frac{\alpha}{2} h(p s, q r)\right)=\left(\frac{\alpha}{2} h(p, q)\right)\left(\frac{\alpha}{2} h(r, s)\right)
$$

which reduces to

$$
h(p r, q s)+h(p s, q r)=h(p, q) h(r, s)
$$

for all $p, r, q, s \in G$.

From Theorem 2.2 we now have that if there is a $c \in Z(G)$ such that $2 h\left(c^{2}, e\right)=h(c, e)^{2}$, then

$$
2 h(c, x)=h(c, e) h(x, e)
$$

for all $x \in G$. Using the definition of $h$ we get that if there is a $c \in Z(G)$ such that

$$
\begin{aligned}
2\left(\frac{2}{\alpha}\right) g\left(c^{2}, e\right) & =\left(\frac{2}{\alpha} g(c, e)\right)^{2} \\
g\left(c^{2}, e\right) & =\frac{1}{\alpha} g(c, e)^{2}
\end{aligned}
$$

then

$$
\begin{aligned}
2\left(\frac{2}{\alpha}\right) g(c, x) & =\left(\frac{2}{\alpha} g(c, e)\right)\left(\frac{2}{\alpha} g(x, e)\right) \\
g(c, x) & =\frac{1}{\alpha} g(c, e) g(x, e)
\end{aligned}
$$

for all $x \in G$ and $c \in Z(G)$. This completes the first case.

If there is a $c \in Z(G)$ such that $g\left(c^{2}, e\right) \neq \frac{1}{\alpha} g(c, e)^{2}$ then there exists two distinct characters $m_{1}, m_{2}$ on $G$ such that

$$
h(p, r)=m_{1}(p) m_{2}(r)+m_{2}(p) m_{1}(r)
$$


for all $p, r \in G$. Therefore, from the definition of $h$

$$
g(p, r)=\frac{\alpha}{2}\left(m_{1}(p) m_{2}(r)+m_{2}(p) m_{1}(r)\right)
$$

for all $p, r \in G$. From that way that $f$ is defined in terms of $g$ we get that

$$
f(p, r)=\frac{\alpha^{2}}{4}\left(m_{1}(p) m_{2}(r)+m_{2}(p) m_{1}(r)\right)
$$

for all $p, r \in G$. This completes the second case and thus completes the proof. 


\section{CHAPTER 5 CONCLUSION}

\subsection{Conclusion and Future Plans}

The purpose of this dissertation has been to study and generalize the solutions of several functional equations defined on the real line to an abstract group. We have made great progress in generalizing several functional equations related to stochastic distance measures. New methods have been created and considered while old techniques and methods have been employed.

In regards to the linear equation, $f(p r, q s)+g(p s, q r)=h(p, q)+k(r, s)$, we have succeeded in finding the general solution on an arbitrary group. We have shown that the solution to the above functional equation when defined on an arbitrary group is similar to the solution when defined on the real line. Several examples were given in the case that all four functions were the same. It would be nice to find more examples, specifically for the generalizations to more than one function.

In regards to the non-linear equation, $f(p r, q s)+f(p s, q r)=f(p, q) f(r, s)$, we have shown that the solution on an arbitrary group is only similar to the solution on the real line under specific conditions. Those conditions are related to whether or not the function is abelian and how the function acts on the center of the group. Since no information is given when the group is centerless it may be worth while to attempt to study this equation on a centerless group.

There are many more things to continue considering with this equation. It 
is my hope that here are other methods that may help us extract more information from the equation. When considering the generalizations of the non-linear functional equation we have not yet been able to solve nor give the structure of the solution when the functions on the left hand side are not the same, i.e. $f(p r, q s)+g(p s, q r)=$ $f(p, q) f(r, s)$. We continue to work to find new methods to extend the study to four different functions.

Recently, the non-linear equation has been considered by Stetækr [15] when some of the variables are switched, that is the functional equation $f(p r, q s)+$ $f(s p, r q)=f(p, q) f(r, s)$. He was able to find the solution on a semigroup. It happens to be a corollary to the solution of the functional equation $f(x y)+f(\sigma(y) x)=$ $2 f(x) f(y)$, where $\sigma$ is a homomorphism. By letting $x=(p, q), y=(r, s)$, and letting $\sigma$ be a function that swaps variables one can see how the two are related. Similarly, we would like to study the functional equation $f(p r, q s)+f(s p, r q)=$ $g(p, q) h(r, s)$ by considering the functional equation $f(x y)+f(\sigma(y) x)=g(x) h(y)$ on a group or a semigroup.

It may be of interest to consider the generalized linear functional equation with a switch of variables, that is $f(p r, q s)+g(s p, r q)=h(p, q)+k(r, s)$. It can be shown that the case in which all four functions are the same is a corollary to Theorem 2.2 and hence several generalizations follow. Using the previously stated technique on the linear functional equation the functional equation $f(x y)+f(\sigma(y) x)=2 f(x)+$ $2 f(y)$ arises. Based upon some results of $\mathrm{Ng}$ and Zhao [8] it may be possible to solve this on a free group, a symmetric group, and a few other specific types of groups. Finding the solution on an arbitrary group may prove to be a bit more of a challenge.

I have recently begun to consider a combination of the linear and non-linear equations, that being the functional equation $f(p r, q s)+f(p s, q r)=2 f(p, q)+$ $2 f(r, s)+f(p, q) f(r, s)$. I have been able to solve this along with a few other 
generalizations. Ideally, I would like to study the functional equation $f(p r, q s)+$ $g(p s, q r)=2 h(p, q)+2 k(r, s)+j(p, q) t(r, s)$.

Hopefully the future work in this area will be as rewarding and fruitful as this work has been! 


\section{REFERENCES}

[1] J. K. Chung, Pl. Kannappan, and C. T. Ng, A generalization of the cosine-sine functional equation on groups, Linear Algebra Appl. 66 (1985), 259-277.

[2] J. K. Chung, Pl. Kannappan, C. T. Ng, and P. K. Sahoo, Measures of distance between probability distributions, J. Math. Anal. Appl. 139 (1989), 280-292.

[3] L. Corovei, On some functional equations for the homomorphisms, Bul. St. Inst. Polit. Cluj-Napoca, Series Mat. Flz. Aec. Ap. 22 (1979), 14-19.

[4] H. Hunt and P. K. Sahoo, On a nonlinear functional equation on groups associated with the characterization of stochastic distance measures, Submitted.

$[5] \_$, On a functional equation on groups arising from the characterization of stochastic distance measures, Aequationes Math. (October 2013), Available Online, DOI: 10.1007/s00010-013-0231-9.

[6] Pl. Kannappan, P. K. Sahoo, and J. K. Chung, An equation associated with the distance between probability distributions, Ann. Math. Silesianal 8 (1994), $39-58$.

[7] S. Kullback and R. A. Leibler, On information and sufficiency, Annals. Math. Statist. 22 (1951), 79-86.

[8] C. T. Ng and H. Y. Zhao, Kernel of the second order Cauchy difference on groups, Aequationes Math. 86 (2013), 155-170. 
[9] T. Riedel and P. K. Sahoo, On a generalization of a functional equation associated with the distance between the probability distributions, Publ. Math. Debrecen 46 (1995), 125-135.

[10] _ On two functional equations connected with the characterizations of the distance measures, Aequationes Math. 54 (1997), 125-135.

[11] P. K. Sahoo, On a functional equation associated with stochastic distance measures, Bull. Korean Math. Soc. 36 (1999), 287-303.

[12] P. K. Sahoo and Pl. Kannappan, Introduction to functional equations, Chapman and Hall/CRC, 2011.

[13] P. K. Sahoo and T. Riedel, Mean value theorems and functional equations, World Scientific Publishing Co Inc, 1999.

[14] H. Stetkær, Functional equations on groups, World Scientific Publishing, Singapore, 2013.

[15] _ A variant of d'Alembert's functional equation, Aequationes Math. (2014).

[16] D. Yang, The symmetrized sine addition formula, Aequationes Math. 82 (2011), 299-318. 


\section{CURRICULUM VITAE}

\section{Heather B. Hunt}

hbhunt2@gmail.com

hbhunt01@louisville.edu

\section{EDUCATION}

University of Louisville, Louisville, $K Y$

Ph.D. in Applied and Industrial Mathematics

June 25th, 2014

Areas of Concentration: Functional Equations and Group Theory

Focus of Dissertation: Solving functional equations on non-commutative groups using ideas from analysis, abstract algebra, calculus, and differential equations

University of Louisville, Louisville, $K Y$

M.A. Mathematics

December 2011

University of Kentucky, Lexington, $K Y$

B.S. Mathematics

May 2009

Minor in Statistics, Graduated Summa Cum Laude

\section{TEACHING EXPERIENCE}

University of Louisville, Louisville $K Y$

Graduate Teaching Assistant Mathematics

2009-Present

Primary Instructor for the following courses: 
- MATH 105: Contemporary Mathematics in Summer 2011 and Summer 2012 Help students understand how to use mathematics to solve concrete problems in the modern world; covering topics such as interest, payments, linear programming, and voting theory and apportionment

- MATH 190: Pre-Calculus in Spring 2013 and Fall 2013

Help students to develop critical thinking skills and algebraic skills that are required in calculus; master the ability to solve equations and inequalities involving rational, exponential and logarithmic equations; and become proficient in the use of trigonometric functions.

- MATH 205: Calculus in Summer 2013

Help students to learn to calculate limits both graphically and analytically, recognize the definition of the derivative as a limit and identify the relationship between derivatives and graphs of functions, describe the definition of the definite integral as a limit of Riemann sums and interpret the definition as an area; understand the relationship between the definite integral and antiderivatives via the fundamental theorem of calculus, and master the standard formulas for computing derivatives and anti-derivatives of functions.

Teaching Assistant for the following courses:

- MATH 105: Contemporary Mathematics

- MATH 107: Finite Mathematics

- MATH 109: Elementary Statistics

- MATH 111: College Algebra

\section{RELATED EXPERIENCE}


Private, Variable Location

Tutor

2009-Present

Help elementary school, middle school, high school and undergraduate students to comprehend their current course material, further their knowledge and understanding of the concepts, and review past material to ensure a strong background in the following subject areas: Pre-Algebra, Algebra I and II, Geometry, Pre-Calculus, College Algebra, and Calculus I and II.

Department of Mathematics, University of Kentucky, Lexington, KY Undergraduate Assistant

2008-2009

Assist professors in proctoring exams and grading, hold office hours to answer students' questions, and work closely with the math department to help students succeed in lower level undergraduate mathematics courses.

The Study, University of Kentucky, Lexington, $K Y$

Academic Enhancement

2006-2009

Work with students on algebra, calculus, and finite mathematics, assist students with problem solving skills, create a friendly and welcoming environment for students, act as a mentor to guide students in the right path while allowing them to make their own decisions.

\section{PAPERS AND PUBLICATIONS}

- Jaeyoung Chung, Heather Hunt, Allison Perkins, and Prasanna Sahoo, "Stability of a simple Levi-Civitá functional equation on non-unital commutative semigroups", Proceedings Mathematical Sciences, Indian Academy of Sciences Accepted for publication, May 2013

- Heather B. Hunt and Prasanna K. Sahoo, "On a functional equation on groups 
arising from the characterization of stochastic distance measures", Aequationes Mathematicae

Available Online, October 2013

- Heather B. Hunt and Prasanna K. Sahoo, "On a non-linear functional equation on groups associated with the characterization of stochastic distance measures" Submitted

\section{PRESENTATIONS}

- "Several Examples of A Functional Equation Defined on Groups", 2014 Annual KYMAA Meeting, Murray State University, Murray, KY, March 28th, 2014

- "A Generalized Linear Functional Equation Defined on Groups", National Joint Mathematics Meeting, Baltimore, MD, January 16th, 2014

- "A Linear Functional Equation on Groups", Central Sectional Joint Mathematics Meeting, Washington University, St. Louis, MO, October 19th, 2013

- "Some Functional Equations on Groups Arising from Stochastic Distance Measures", Candidacy Examination, University of Louisville, Louisville, KY, August 28th, 2013

- "An Introduction to Latex", Research Methods in Mathematics Course, University of Louisville, Louisville, KY, May 29, 2013

- "A Functional Equation from Stochastic Distance Measure", 2013 Annual KYMAA Meeting, Transylvania University, Lexington, KY, April 5, 2013

- "A Functional Equation on Groups", 32nd Annual WKU Mathematics Symposium, Western Kentucky University, Bowling Green, KY, October 13, 2012 


\section{LEADERSHIP/ MENTORING}

Department of Mathematics, University of Louisville, Louisville, KY

Peer Mentor for Mathematics Graduate Students

2012-Present

Help new graduate students feel welcome and become a part of the Mathematics Department community, attempt to answer any questions that students may have or direct them to someone who can answer their questions, create and maintain a bulletin board informing mathematics graduate students about upcoming events, conferences, funding opportunities, etc.; organize and plan a departmental mock interview for students; plan a picnic at the end of the academic year hosted by the graduate students for all faculty and staff in the Mathematics Department.

Department of Mathematics, University of Louisville, Louisville, KY

Assistant to professor for the purpose of coordinating the American Mathematical Society Sectional Meeting

Fall 2013

Recruit, organize, and schedule volunteers to direct visitors around campus and run the registration desk, create signs to direct visitors to parking and different locations on the University of Louisville's campus, and work with a company to have the signs printed professionally by a given deadline.

University of Louisville, Louisville, $K Y$

Graduate Student Council Department Representative 2012-Present Attend monthly meetings, share the needs and concerns of the graduate students within my department with others, occasionally fill in for representatives at the graduate student senate meetings to vote on important items affecting the University's graduate students, and relay all important information about upcoming events, changes, and funding. 
University of Louisville, Louisville, $K Y$

Graduate Student Union Department Representative 2012-Present Attend monthly meetings, open the lines of communication between graduate students in the Department of Mathematics and graduate students in other departments, assist in organizing family friendly graduate student events, and relay all important information about upcoming events, changes, and funding.

AWARDS

- Scholar Athlete Faculty Mentor, University of Louisville

March 2014

- Graduate Student Spotlight Nominee, University of Louisville January 2014

- Service to Graduate Student Council, University of Louisville 2013 and 2014

- Teaching Assistantship, University of Louisville

2009-Present

- Robert B. Royster Award, University of Kentucky

2009

- JC Eaves Scholarship, University of Kentucky 2007 